\title{
Identification of speculative bubbles using state-space models with Markov-switching
}

Nael Al-Anaswah and Bernd Wilfling ${ }^{\dagger}$

$3 / 2009$

${ }^{\dagger}$ Department of Economics, University of Münster, Germany 


\title{
Identification of speculative bubbles using state-space models with Markov-switching
}

\author{
Nael Al-Anaswah ${ }^{a}$, Bernd Wilfling ${ }^{\mathrm{a}, *}$ \\ ${ }^{a}$ Westfälische Wilhelms-Universität Münster, Department of Economics, Am \\ Stadtgraben 9, 48143 Münster, Germany
}

(Date of this version: September 21, 2009)

\begin{abstract}
In this paper we use a state-space model with Markov-switching to detect speculative bubbles in stock-price data. Our two innovations are (1) to adapt this technology to the state-space representation of a well-known present-value stock-price model, and (2) to estimate the model via Kalman-filtering using a plethora of artificial as well as real-world data sets that are known to contain bubble periods. Analyzing the smoothed regime probabilities, we find that our technology is well suited to detecting stock-price bubbles in both types of data sets.
\end{abstract}

JEL-classification codes: C22; G12

Key words: Stock market dynamics; Detection of speculative bubbles; Present value models; State-space models with Markov-switching

\footnotetext{
${ }^{*}$ Corresponding author. Phone: +49 - 251 - 83 - 25040, Fax: +49 - 251 -83 - 25042, e-mail address: bernd.wilfling@wiwi.uni-muenster.de
} 


\section{Introduction}

A classical belief is that under rational expectations and rational behaviour of economic agents any asset should be priced according to its market fundamental value. In line with this view, a persistent and substantial divergence between an asset price and its fundamental value is often regarded as market irrationality. However, recent work elaborates why the dynamics of an asset price may well contain a self-fulfilling bubble component and that the explosive asset-price behaviour caused by the bubble may be consistent with rational behaviour among market participants.

Up to date, a multitude of theoretical studies have examined the emergence of (stock market) bubbles and their structural properties under rational expectations (see, for instance, Tirole, 1982; Allen et al., 1993; Allen and Gale, 2000; Abreu and Brunnermeier, 2003). A closely related strand of literature is concerned with the econometric detection of speculative bubbles. These papers can roughly be divided into two groups. The first group of studies is based on so-called indirect bubble tests. Here, the authors apply sophisticated cointegration and unit-root tests to a dividend-price relationship and try to overcome the well-known econometric weaknesses of the standard tests. Frequently cited articles belonging to this category include Diba and Grossman (1988), Evans (1991) and several other contributions cited in McMillan (2007). The second group of studies, which is the more relevant for our paper, implement direct tests for speculative bubbles by explicitly formulating the existence of a bubble in the alternative hypothesis. Examples of such direct test procedures are West (1987) and Wu (1997).

The key idea of West's (1987) direct bubble test is to compare two alternative estimators (i.e. an indirect and a direct estimator) for one particular parameter. More concretely, the indirect estimator is constructed from two different estimations, namely (1) from the estimation of the observable no-bubble Euler equation, and (2) from the estimation of a stationary autoregressive process which is assumed to govern dividends. Both estimations can be combined to yield an indirect estimate of the linear relationship between dividends and stock prices. Alternatively, the linear relationship between dividends and stock prices can be estimated directly by performing a straightforward linear regression of stock prices on dividends. Under the null hypothesis of 'no bubble', the direct and the indirect estimates of the linear relationship should be equal (within the limits of statistical accuracy) while under the alternative of 'a rational bubble' both estimates should differ significantly from each other. Hence, the basic idea of West's (1987) test is to interpret a statistically significant difference between the direct and the indirect estimates as an indication of a speculative bubble. This interpretation 
may be strengthened further by additionally applying specification tests to the Euler equation and the autoregressive representation of dividends in the hope of ruling out all model misspecification and leaving bubbles as the only possible source of the discrepancy between the two estimates.

Essentially, West's procedure tests the standard present value model against an unspecified alternative which is interpreted as having emerged from a speculative bubble. However, the test does not generate a time series of the bubble component. By contrast, $\mathrm{Wu}$ (1997), who also considers the deviation of stock prices from the present value model, uses the discrepancies to construct a bubble time series. As in West (1987) he assumes that dividends follow an autoregressive process and treats the bubble as an unobservable variable which he estimates using the Kalman filter. In so doing, Wu finds that large portions of stock-price movements within the S\&P 500 may be ascribed to speculative bubbles.

Another class of econometric models, which have been used intensively for the detection of bubble components, are so-called Markov-switching (or regime-switching) models. These models are designed to capture discrete shifts in the generating process of time series data and were introduced by Hamilton (1988, 1989). An important application of Markov-switching models in the bubble literature is presented by Hall et al. (1999) who treat each component of a simulated bubble process as a separate Markov regime with constant transition probabilities between the regimes. Within a Monte Carlo experiment they analyze the power of Augmented-Dickey-Fuller unitroot tests with Markov-switching (Markov-switching ADF tests) and apply these test procedures to detect bubble episodes. Although this methodology may be criticized on econometric grounds (see for example Vigfusson and van Norden, 1998), Markovswitching approaches constitute a useful tool for modeling bubbles that switch between two or more states (see Driffill and Sola, 1998; Brooks and Katsaris, 2005).

In this paper, we treat the bubble as an unobservable variable as in $\mathrm{Wu}$ (1997) but extend his framework by allowing the bubble to switch between alternative regimes. Through this, we aim at separating the moderately growing from the explosive period in the bubble process. Technically speaking, we implement regime-switching in our unobserved-components framework by adopting an econometric technology that was first mentioned by Harrison and Stevens (1976) and was given a more thorough formal treatment by Gordon and Smith (1988). Kim and Nelson (1999) embed the methodology in a more accessible framework by showing how to use state-space models that are subject to regime-switching. Up to now, this econometric technique has mainly been used for the detection of turning points in business-cycle research (see for example 
Chauvet, 1998; Chauvet and Piger, 2003; Chauvet and Hamilton, 2006). However, the application to the bubble literature is still lacking and constitutes the innovation of our paper.

In line with several previous studies from the bubble literature, we analyze both artificially generated bubble data as well as real-world data sets. The inclusion of artificial bubble processes has the advantage of knowing exactly when a bubble starts to evolve over time. Thus, we obtain precise information on the statistical quality of our bubble-detection method. By contrast, the identification of bubble periods in real-world data sets turns out to be a more complicated matter. For this data type we are reliant on what economic historians have classified ex-post as bubble periods. In our empirical analysis below, we rely on the work of Kindleberger and Aliber (2005) who classify bubble periods in real-world stock-market data.

Our study has two major findings. First, we show that Markov-switching in the data-generating process of real-world stock-price bubbles appears to be a statistically significant phenomenon. Second, we obtain the encouraging overall result that our econometric framework is able to detect most bubble periods in our artificial data sets and is even more successful in tracking down real-world stock-price bubbles as classified by Kindleberger and Aliber (2005).

The remainder of the paper is organized as follows. Section 2 briefly reviews the basic present value model. Section 3 transforms the present value model into a state-space representation. We demonstrate how the state-space model including the unobserved asset-price bubble can be estimated by the Kalman filter. In Section 4 we incorporate Markov-switching elements into the state-space model. Section 5 describes our artificial bubble processes, our real-world data sets and presents the estimation results. Section 6 offers some concluding remarks.

\section{Economic Model}

In this section we briefly review the standard present-value model of stock prices on the basis of the log-linear approximation as suggested by Campbell and Shiller (1988a,b). For this, consider the following rational-expectations model of stock-price determination:

$$
q=\kappa+\psi E_{t}\left(p_{t+1}\right)+(1-\psi) d_{t}-p_{t},
$$

where $q$ is the required log gross return rate, $E_{t}(\cdot)$ is the mathematical expectation operator conditional on all information available at date $t, p_{t} \equiv \ln \left(P_{t}\right)$ is the $\log$ real stock price at date $t, d_{t} \equiv \ln \left(D_{t}\right)$ is the log real dividend paid at date $t$ and $\kappa$ and $\psi$ 
$(0<\psi<1)$ are parameters of linearization. ${ }^{1}$

Equation (1) constitutes a linear difference equation for the log stock price which can be routinely solved by forward iteration. Imposing the transversality condition

$$
\lim _{i \rightarrow \infty} \psi^{i} E_{t}\left(p_{t+i}\right)=0
$$

we obtain the unique no-bubble solution (denoted by $p_{t}^{f}$ ) to Eq. (1):

$$
p_{t}^{f}=\frac{\kappa-q}{1-\psi}+(1-\psi) \sum_{i=0}^{\infty} \psi^{i} E_{t}\left(d_{t+i}\right)
$$

The no-bubble solution $p_{t}^{f}$ in Eq. (2) represents the well-known present-value relation stating that the log stock price is equal to the present value of expected future log dividends. However, it is important to note that from a mathematical point of view the above transversality condition may not be satisfied. In that case, the no-bubble solution $p_{t}^{f}$ represents only a particular solution to the difference equation (1), the general solution of which has the form

$$
p_{t}=p_{t}^{f}+B_{t}
$$

with the process $\left\{B_{t}\right\}$ satisfying the homogeneous difference equation

$$
E_{t}\left(B_{t+i}\right)=\frac{B_{t}}{\psi^{i}} \quad \text { for } i=1,2, \ldots
$$

(see for example Cuthbertson and Nitzsche, 2004, pp. 397-401).

Obviously, the general solution in Eq. (3) consists of two components. First, the no-bubble solution $p_{t}^{f}$ only depends on $\log$ dividends and is therefore often termed the market-fundamental solution. Second, the mathematical entity $B_{t}$ may be driven by events extraneous to the market and is thus referred to as the rational speculative bubble component.

In order to circumvent nonstationarity problems, it is convenient to express the model in first-difference form which, by virtue of the Eqs. (2) and (3), is given by

$$
\Delta p_{t}=\Delta p_{t}^{f}+\Delta B_{t}=(1-\psi) \sum_{i=0}^{\infty} \psi^{i}\left[E_{t}\left(d_{t+i}\right)-E_{t-1}\left(d_{t+i-1}\right)\right]+\Delta B_{t}
$$

Following $\mathrm{Wu}$ (1997), we also assume that log dividends may contain a unit root but

\footnotetext{
${ }^{1}$ In particular, $\psi$ is the average $\log$ dividend-price ratio and $\kappa$ is defined by $\kappa \equiv-\ln (\psi)-(1-$ $\psi) \ln (1 / \psi-1)$.
} 
that the dividend process $\left\{d_{t}\right\}$ can be approximated by an autoregressive integrated moving average process. In particular, we assume an $\operatorname{ARIMA}(h, 1,0)$ process of the form

$$
\Delta d_{t}=\mu+\sum_{j=1}^{h} \phi_{j} \Delta d_{t-j}+\delta_{t},
$$

with $\delta_{t} \sim N\left(0, \sigma_{\delta}^{2}\right)$ denoting a Gaussian white-noise error term and where the autoregressive order $h$ can be estimated from the data.

In what follows, it is convenient to express the autoregressive process (6) in companion form. Defining the $(h \times 1)$ vectors

$$
\mathbf{y}_{t}=\left(\Delta d_{t}, \Delta d_{t-1}, \ldots, \Delta d_{t-h+1}\right)^{\prime}, \quad \mathbf{u}=(\mu, 0,0, \ldots, 0)^{\prime}, \quad \boldsymbol{\nu}_{t}=\left(\delta_{t}, 0,0, \ldots, 0\right)^{\prime}
$$

and the $(h \times h)$ matrix

$$
\mathbf{A}=\left(\begin{array}{cccccc}
\phi_{1} & \phi_{2} & \phi_{3} & \ldots & \phi_{h-1} & \phi_{h} \\
1 & 0 & 0 & \ldots & 0 & 0 \\
0 & 1 & 0 & \ldots & 0 & 0 \\
\ldots & \ldots & \ldots & \ldots & \ldots & \ldots \\
0 & 0 & 0 & \ldots & 1 & 0
\end{array}\right)
$$

we may write Eq. (6) in the form

$$
\mathbf{y}_{t}=\mathbf{u}+\mathbf{A y}_{t-1}+\boldsymbol{\nu}_{t}
$$

Based on this representation, it follows from Campbell and Shiller (1987) that the solution to our stock-price model (5) can be calculated from the formula

$$
\Delta p_{t}=\Delta d_{t}+\mathbf{m} \Delta \mathbf{y}_{t}+\Delta B_{t}
$$

where $\mathbf{m}$ is an $(h \times 1)$ vector defined as

$$
\mathbf{m}=\mathbf{g A}(\mathbf{I}-\mathbf{A})^{-1}\left[\mathbf{I}-(1-\psi)(\mathbf{I}-\psi \mathbf{A})^{-1}\right],
$$

while the $(h \times 1)$ vector $\mathbf{g}$ is given by $\mathbf{g}=(1,0,0, \ldots, 0)^{\prime}$ and $\mathbf{I}$ symbolizes the $(h \times h)$ identity matrix.

In line with $\mathrm{Wu}(1997)$, we also assume a linear bubble process $\left\{B_{t}\right\}$. Hence, Eq. (4) implies

$$
B_{t}=(1 / \psi) B_{t-1}+\eta_{t},
$$

where the innovation process $\left\{\eta_{t}\right\}$ is assumed to be i.i.d. $N\left(0, \sigma_{\eta}^{2}\right)$. Additonally, we 
assume that $\eta_{t}$ is uncorrelated with the dividend innovation $\delta_{t}$ from Eq. (6).

When estimating the stock-price equation (8), we are confronted with the problem that the bubble component $\left\{B_{t}\right\}$ is unobservable. This fact suggests using a Kalman filtering technique for the application of which we have to express our present-value model in state-space form.

\section{State-space representation and the Kalman filter}

We now express our present-value model in state-space form so that the Kalman filter can be used to estimate the unobservable asset-price bubble. The approach of this section closely follows $\mathrm{Wu}(1995)$.

\subsection{The state-space representation}

Let $\boldsymbol{\beta}_{t}$ be an $(n \times 1)$ vector of unobserved variables referred to as state variables and let $\mathbf{g}_{t}$ and $\mathbf{z}_{t}$ be $(m \times 1)$ and $(l \times 1)$ vectors of observable variables referred to as input and output variables, respectively. Then, the state-space model can be written as

$$
\begin{aligned}
\boldsymbol{\beta}_{t} & =\mathbf{F} \boldsymbol{\beta}_{t-1}+\boldsymbol{\xi}_{t}, \\
\mathbf{z}_{t} & =\mathbf{H} \boldsymbol{\beta}_{t}+\mathbf{D g}_{t}+\boldsymbol{\zeta}_{t},
\end{aligned}
$$

where $\boldsymbol{\xi}_{t}$ and $\boldsymbol{\zeta}_{t}$ are $(n \times 1)$ and $(l \times 1)$ vectors of disturbances and $\mathbf{F}, \mathbf{H}$ and $\mathbf{D}$ are constant real matrices of conformable dimensions. It is assumed that the disturbance vectors $\boldsymbol{\xi}_{t}$ and $\boldsymbol{\zeta}_{t}$ are serially uncorrelated, uncorrelated with each other and that

$$
\begin{gathered}
E\left(\boldsymbol{\xi}_{t}\right)=\mathbf{0}, \quad E\left(\boldsymbol{\zeta}_{t}\right)=\mathbf{0}, \\
E\left(\boldsymbol{\xi}_{t} \boldsymbol{\xi}_{t}^{\prime}\right)=\boldsymbol{\Omega}, \quad E\left(\boldsymbol{\zeta}_{t} \boldsymbol{\zeta}_{t}^{\prime}\right)=\mathbf{R} .
\end{gathered}
$$

The Eqs. (11) and (12) are called transition and measurement equation, respectively.

Basically, our economic model from Section 2 consists of the following three components: the $\operatorname{ARIMA}(h, 1,0)$ dividend process $\left\{\Delta d_{t}\right\}$ from Eq. (6), the stock-price process $\left\{\Delta p_{t}\right\}$ from Eq. (8) and the bubble process $\left\{B_{t}\right\}$ from Eq. (10). It is easy to check, that our complete economic model can be written in state-space form as follows:

$$
\begin{gathered}
\boldsymbol{\beta}_{t}=\left(B_{t}, B_{t-1}\right)^{\prime}, \quad \mathbf{z}_{t}=\left(\Delta d_{t}, \Delta p_{t}\right)^{\prime}, \quad \mathbf{g}_{t}=\left(1, \Delta d_{t}, \Delta d_{t-1}, \Delta d_{t-2}, \ldots, \Delta d_{t-h}\right)^{\prime}, \\
\boldsymbol{\xi}_{t}=\left(\eta_{t}, 0\right)^{\prime}, \quad \boldsymbol{\zeta}_{t}=\left(\delta_{t}, 0\right)^{\prime},
\end{gathered}
$$




$$
\mathbf{F}=\left(\begin{array}{cc}
1 / \psi & 0 \\
1 & 0
\end{array}\right), \quad \mathbf{H}=\left(\begin{array}{cc}
0 & 0 \\
1 & -1
\end{array}\right)
$$

and

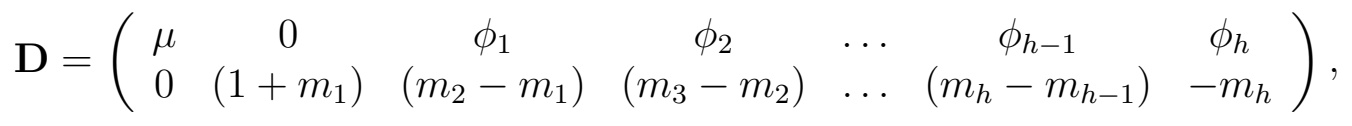

where $m_{i}$ is the $i$ th component of the $(h \times 1)$ vector $\mathbf{m}$ defined in (9). The covariance matrices $\Omega$ and $\mathbf{R}$ are given by

$$
\boldsymbol{\Omega}=\left(\begin{array}{cc}
\sigma_{\eta}^{2} & 0 \\
0 & 0
\end{array}\right) \quad \text { and } \quad \mathbf{R}=\left(\begin{array}{cc}
\sigma_{\delta}^{2} & 0 \\
0 & 0
\end{array}\right)
$$

All in all, our state-space representation treats the asset-price bubble as an unobservable state variable and specifies two transition and two measurement equations. Both transition equations represent the bubble process (10) while the first measurement equation represents the dividend process (6) and the second measurement equation the price process (8).

\subsection{The Kalman filtering technique}

In this section, we outline the Kalman filter procedure with the ultimate aim of estimating stochastic asset-price bubbles. The Kalman filter is extensively discussed in the control literature (see among others Hamilton, 1994a,b).

Our basic problem consists in estimating the unobserved state vector $\boldsymbol{\beta}_{t}$. Let $\boldsymbol{\beta}_{t \mid \tau}$ denote the best linear mean-squared estimate of $\boldsymbol{\beta}_{t}$ given the model and all observed data up to time $\tau$. $\boldsymbol{\beta}_{t \mid \tau}$ and its covariance matrix can then be obtained via the following equations:

$$
\begin{aligned}
\boldsymbol{\beta}_{t \mid t-1} & =\mathbf{F} \boldsymbol{\beta}_{t-1 \mid t-1} \\
\mathbf{P}_{t \mid t-1} & =\mathbf{F P}_{t-1 \mid t-1} \mathbf{F}^{\prime}+\boldsymbol{\Omega}, \\
\boldsymbol{\zeta}_{t \mid t-1} & =\mathbf{z}_{t}-\mathbf{H} \boldsymbol{\beta}_{t \mid t-1}-\mathbf{D} \mathbf{g}_{t} \\
\mathbf{K}_{t} & =\mathbf{P}_{t \mid t-1} \mathbf{H}^{\prime}\left[\mathbf{H} \mathbf{P}_{t \mid t-1} \mathbf{H}^{\prime}+\mathbf{R}\right]^{-1}, \\
\boldsymbol{\beta}_{t \mid t} & =\boldsymbol{\beta}_{t \mid t-1}+\mathbf{K}_{t} \boldsymbol{\zeta}_{t \mid t-1}, \\
\mathbf{P}_{t \mid t} & =\left[\mathbf{I}-\mathbf{K}_{t} \mathbf{H}\right] \mathbf{P}_{t \mid t-1},
\end{aligned}
$$

where $\mathbf{P}_{t \mid t-1}=E\left[\left(\boldsymbol{\beta}_{t}-\boldsymbol{\beta}_{t \mid t-1}\right)\left(\boldsymbol{\beta}_{t}-\boldsymbol{\beta}_{t \mid t-1}\right)^{\prime}\right]$ and $\mathbf{P}_{t \mid t}=E\left[\left(\boldsymbol{\beta}_{t}-\boldsymbol{\beta}_{t \mid t}\right)\left(\boldsymbol{\beta}_{t}-\boldsymbol{\beta}_{t \mid t}\right)^{\prime}\right]$ are the error covariance matrices for $1 \leq t \leq T$. The above equations form the Kalman filter 
and are computed forward recursively. More efficient estimates of the state vector and its error covariance matrix can be obtained by using all information up to time $T$ via the following full-sample smoother:

$$
\begin{aligned}
\boldsymbol{\beta}_{t-1 \mid T} & =\boldsymbol{\beta}_{t-1 \mid t-1}+\mathbf{J}_{t-1}\left(\boldsymbol{\beta}_{t \mid T}-\mathbf{F} \boldsymbol{\beta}_{t \mid t-1}\right) \\
\mathbf{P}_{t-1 \mid T} & =\mathbf{P}_{t-1 \mid t-1}+\mathbf{J}_{t-1}\left(\mathbf{P}_{t \mid T}-\mathbf{P}_{t \mid t-1}\right) \mathbf{J}_{t-1}^{\prime}, \\
\mathbf{J}_{t-1} & =\mathbf{P}_{t-1 \mid t-1} \mathbf{F}^{\prime} \mathbf{P}_{t \mid t-1}^{-1}, t=T-1, T-2, \ldots, 1 .
\end{aligned}
$$

This smoother is run backward recursively.

The Kalman filter treats the model parameters as known. In practice, however, the parameter matrices $\mathbf{F}, \mathbf{H}, \mathbf{D}, \boldsymbol{\Omega}$ and $\mathbf{R}$ are unknown and need to be estimated. Collecting all unknown parameters in the vector $\boldsymbol{\alpha}$, we can estimate all parameters by maximising the following log likelihood function (see Hamilton, 1994a, pp. 385-86):

$$
\begin{aligned}
L(\boldsymbol{\alpha} \mid \mathbf{z}, \mathbf{g})=\mathrm{const}-\frac{1}{2} \sum_{t=1}^{T}( & \ln \left[\operatorname{det}\left(\mathbf{H P}_{t \mid t-1} \mathbf{H}^{\prime}+\mathbf{R}\right)\right] \\
& \left.+\boldsymbol{\zeta}_{t \mid t-1}^{\prime}\left(\mathbf{H P}_{t \mid t-1} \mathbf{H}^{\prime}+\mathbf{R}\right)^{-1} \boldsymbol{\zeta}_{t \mid t-1}\right)
\end{aligned}
$$

In Eq. (16) the innovation $\boldsymbol{\zeta}_{t \mid t-1}$ and the error covariance matrix $\mathbf{P}_{t \mid t-1}$ are both implicit functions of the unknown parameter vector $\boldsymbol{\alpha}$ and are evaluated using the Kalman filter. Once the maximum-likelihood estimate of $\boldsymbol{\alpha}$ has been obtained, the smoothed estimates of the state vector and its error covariance matrix can be determined by the Kalman filter and the full-sample smoother stated above.

\section{State-space models with Markov-switching}

We now introduce distinct regimes into the state-space model from the previous section. The idea behind this is that alternative regimes might enable us to distinguish between moderately growing and explosive periods in bubble processes (see Evans, 1991). In this paper, we restrict attention to the modeling of two distinct regimes. The econometric exposition of this section closely follows Kim and Nelson (1999, Chapter 5) who also cover the more general case of $M \geq 2$ distinct regimes.

\subsection{Model specification}

We begin with the state-space representation of a dynamic system consisting of the transition and measurement equations (11) and (12). Additionally, we now allow the 
parameters in the matrices $\mathbf{F}, \mathbf{H}, \mathbf{D}, \boldsymbol{\Omega}$ and $\mathbf{R}$ to switch between two distinct regimes and therefore write the state-space model as

$$
\begin{aligned}
\boldsymbol{\beta}_{t} & =\mathbf{F}_{S_{t}} \boldsymbol{\beta}_{t-1}+\boldsymbol{\xi}_{t}, \\
\mathbf{z}_{t} & =\mathbf{H}_{S_{t}} \boldsymbol{\beta}_{t}+\mathbf{D}_{S_{t}} \mathbf{g}_{t}+\boldsymbol{\zeta}_{t}, \\
\left(\begin{array}{c}
\boldsymbol{\xi}_{t} \\
\boldsymbol{\zeta}_{t}
\end{array}\right) & \sim N\left(0,\left(\begin{array}{cc}
\boldsymbol{\Omega}_{S_{t}} & 0 \\
0 & \mathbf{R}_{S_{t}}
\end{array}\right)\right) .
\end{aligned}
$$

The subscripts $S_{t}$ in the above equations are meant to indicate that the parameters in the matrices are governed by an unobservable two-state random variable $S_{t}\left(S_{t}=\right.$ $1,2)$ which determines the regime the parameters are in at date $t$. We specify the probabilistic nature of the regime-indicator $S_{t}$ by a first-order Markov-process with time-invariant transition probabilities $p_{i j}=\operatorname{Pr}\left[S_{t}=j \mid S_{t-1}=i\right]$ which we collect in the transition-probability matrix

$$
\boldsymbol{\Pi}=\left(\begin{array}{cc}
p_{11} & 1-p_{22} \\
1-p_{11} & p_{22}
\end{array}\right) .
$$

In what follows, we use the compact notation $\mathbf{F}_{i}$ when $S_{t}=i$ (and similarly for the matrices $\left.\mathbf{H}_{S_{t}}, \mathbf{D}_{S_{t}}, \Omega_{S_{t}}, \mathbf{R}_{S_{t}}\right)$.

\subsection{The basic filter, model estimation, inference and smooth- ing}

By analogy with the Kalman filtering technique for the single-regime state-space model described in Section 3.2, we assume in a first step that the parameters of the two-regime Markov-switching state-space model (17) to (20) are all known. Let $\boldsymbol{\Psi}_{t-1}$ denote the vector of observations available as of date $t-1$. In the derivation of the Kalman filter for the single-regime state-space model, the main objective is to form a forecast of the unobserved state vector $\boldsymbol{\beta}_{t}$ based on $\boldsymbol{\Psi}_{t-1}$ which we denote by

$$
\boldsymbol{\beta}_{t \mid t-1}=E\left[\boldsymbol{\beta}_{t} \mid \Psi_{t-1}\right] .
$$

Similarly, in the single-regime state-space model from the Sections 3.1 and 3.2 the matrix $\mathbf{P}_{t \mid t-1}$ represents the mean squared error of this forecast and may be written as

$$
\mathbf{P}_{t \mid t-1}=E\left[\left(\boldsymbol{\beta}_{t}-\boldsymbol{\beta}_{t \mid t-1}\right)\left(\boldsymbol{\beta}_{t}-\boldsymbol{\beta}_{t \mid t-1}\right)^{\prime} \mid \boldsymbol{\Psi}_{t-1}\right] .
$$


By contrast, in our state-space model (17) to (20) with Markov-switching parameters our objective is to form a forecast of $\boldsymbol{\beta}_{t}$ which is based not just on $\boldsymbol{\Psi}_{t-1}$, but which is also conditional on the transition of the regime-indicator from state $S_{t-1}=i$ to state $S_{t}=j($ for $i, j=1,2)$ :

$$
\boldsymbol{\beta}_{t \mid t-1}^{(i, j)}=E\left[\boldsymbol{\beta}_{t} \mid \Psi_{t-1}, S_{t}=j, S_{t-1}=i\right]
$$

The Kalman-filter algorithm from the Eqs. (21) to (26) below computes a battery of $2^{2}=4$ such forecasts for each date $t$, namely one forecast for each possible Markovregime combination $(i, j)$. Associated with these four forecasts for date $t$ are the four mean squared error matrices

$$
\mathbf{P}_{t \mid t-1}^{(i, j)}=E\left[\left(\boldsymbol{\beta}_{t}-\boldsymbol{\beta}_{t \mid t-1}\right)\left(\boldsymbol{\beta}_{t}-\boldsymbol{\beta}_{t \mid t-1}\right)^{\prime} \mid \boldsymbol{\Psi}_{t-1}, S_{t}=j, S_{t-1}=i\right]
$$

Conditional on $S_{t-1}=i$ and $S_{t}=j$, the Kalman filter can be stated as follows:

$$
\begin{aligned}
\boldsymbol{\beta}_{t \mid t-1}^{(i, j)} & =\mathbf{F}_{j} \boldsymbol{\beta}_{t-1 \mid t-1}^{i}, \\
\mathbf{P}_{t \mid t-1}^{(i, j)} & =\mathbf{F}_{j} \mathbf{P}_{t-1 \mid t-1}^{i} \mathbf{F}_{j}^{\prime}+\boldsymbol{\Omega}_{j}, \\
\boldsymbol{\zeta}_{t \mid t-1}^{(i, j)} & =\mathbf{z}_{t}-\mathbf{H}_{j} \boldsymbol{\beta}_{t \mid t-1}^{(i, j)}-\mathbf{D}_{j} \mathbf{g}_{t}, \\
\mathbf{f}_{t \mid t-1}^{(i, j)} & =\mathbf{H}_{j} \mathbf{P}_{t \mid t-1}^{(i, j)} \mathbf{H}_{j}^{\prime}+\mathbf{R}_{j}, \\
\boldsymbol{\beta}_{t \mid t}^{(i, j)} & =\boldsymbol{\beta}_{t \mid t-1}^{(i, j)}+\mathbf{P}_{t \mid t-1}^{(i, j)} \mathbf{H}_{j}^{\prime}\left[\mathbf{f}_{t \mid t-1}^{(i, j)}\right]^{-1} \boldsymbol{\zeta}_{t \mid t-1}^{(i, j)}, \\
\mathbf{P}_{t \mid t}^{(i, j)} & =\left(\mathbf{I}-\mathbf{P}_{t \mid t-1}^{(i, j)} \mathbf{H}_{j}^{\prime}\left[\mathbf{f}_{t \mid t-1}^{(i, j)}\right]^{-1} \mathbf{H}_{j}\right) \mathbf{P}_{t \mid t-1}^{(i, j)} .
\end{aligned}
$$

Here, $\boldsymbol{\beta}_{t-1 \mid t-1}^{i}$ is an inference on $\boldsymbol{\beta}_{t-1}$ based on information up to time $t-1$ given $S_{t-1}=i . \quad \boldsymbol{\beta}_{t \mid t-1}^{(i, j)}$ is an inference on $\boldsymbol{\beta}_{t}$ based on information up to time $t-1$ given $S_{t}=j$ and $S_{t-1}=i . \boldsymbol{\zeta}_{t \mid t-1}^{(i, j)}$ is the conditional forecast error of $\mathbf{z}_{t}$ based on information up to time $t-1$ given $S_{t-1}=i$ and $S_{t}=j$. And finally, $\mathbf{f}_{t \mid t-1}^{(i, j)}$ is the conditional variance of the forecast error $\boldsymbol{\zeta}_{t \mid t-1}^{(i, j)}$.

An obvious drawback of the above Kalman-filter algorithm is that in our two-regime specification each iteration produces a 2-fold increase in the number of cases that have to be considered. So, by date $t=10$, for example, 1024 distinct cases were to be considered. Therefore, it appears to be necessary to introduce some approximations in order to make the Kalman filter operable. The key idea to accomplish this is to collapse terms in the right way at the right time by somehow reducing each of the quadruple 
posteriors $\boldsymbol{\beta}_{t \mid t}^{(i, j)}$ and $\mathbf{P}_{t \mid t}^{(i, j)}$ from the Eqs. (25) and (26) to the 2 twofold posteriors $\boldsymbol{\beta}_{t \mid t}^{j}$ and $\mathbf{P}_{t \mid t}^{j}$.

A variety of explicit collapsing procedures have been scattered in the literature (see among others Harrison and Stevens, 1976; Smith and Makov, 1980; Gordon and Smith, 1988). However, in our subsequent empirical implementation we use the approximation approach provided by Kim and Nelson (1999). More precisely, at the end of each iteration we collapse the quadruple posteriors $\boldsymbol{\beta}_{t \mid t}^{(i, j)}$ and $\mathbf{P}_{t \mid t}^{(i, j)}$ to

$$
\boldsymbol{\beta}_{t \mid t}^{j}=\frac{\sum_{i=1}^{2} \operatorname{Pr}\left[S_{t-1}=i, S_{t}=j \mid \boldsymbol{\Psi}_{t}\right] \boldsymbol{\beta}_{t \mid t}^{(i, j)}}{\operatorname{Pr}\left[S_{t}=j \mid \mathbf{\Psi}_{t}\right]}
$$

and

$$
\mathbf{P}_{t \mid t}^{j}=\frac{\sum_{i=1}^{2} \operatorname{Pr}\left[S_{t-1}=i, S_{t}=j \mid \boldsymbol{\Psi}_{t}\right]\left\{\boldsymbol{P}_{t \mid t}^{(i, j)}+\left(\boldsymbol{\beta}_{t \mid t}^{j}-\boldsymbol{\beta}_{t \mid t}^{(i, j)}\right)\left(\boldsymbol{\beta}_{t \mid t}^{j}-\boldsymbol{\beta}_{t \mid t}^{(i, j)}\right)^{\prime}\right\}}{\operatorname{Pr}\left[S_{t}=j \mid \boldsymbol{\Psi}_{t}\right]}
$$

respectively. Apart from that, we also use Kim and Nelson's (1999) 3-step procedure to make inferences on the probability terms which appear on the right-hand side of the collapsing equations $(27)$ and $(28){ }^{2}$

In order to estimate the parameters of our state-space model, we implement the procedure depicted in Figure 5.1 in Kim and Nelson (1999, p. 105). After obtaining the appropriate starting values $\boldsymbol{\beta}_{t \mid t}^{j}, \mathbf{P}_{t \mid t}^{j}$ and $\operatorname{Pr}\left[S_{0}=j \mid \boldsymbol{\Psi}_{t}\right]$, we run the Kalman filter (21) to (26) while collapsing the posteriors $\boldsymbol{\beta}_{t \mid t}^{(i, j)}$ and $\mathbf{P}_{t \mid t}^{(i, j)}$ after the end of each iteration to $\boldsymbol{\beta}_{t \mid t}^{j}$ and $\mathbf{P}_{t \mid t}^{j}$ as given in the Eqs. (27) and (28). As a by-product of the filter, we obtain the (approximate) log likelihood function from which we estimate the model parameters by using a nonlinear maximization technique. ${ }^{3}$

After parameter estimation we can finally make inference about the regime-indicator $S_{t}$ and the unobserved state-vector $\boldsymbol{\beta}_{t}$ by using full-sample information. A variety of different smoothing approaches have been suggested and discussed in the literature (see for example Kitagawa, 1987). In this study we use the 4-step algorithm given in Kim and Nelson (1999, pp. 106-109) to compute the smoothed probabilities $\operatorname{Pr}\left[S_{t}=j \mid \Psi_{T}\right]$ and to derive the smoothed values of the state-vector $\boldsymbol{\beta}_{t \mid T}$ for $t=1,2, \ldots, T{ }^{4}$

Finally, some remarks on the explicit parameters which we estimate in our subsequent empirical analysis are in order. In the most general setting, we could specify all parameters appearing in the matrices $\mathbf{F}, \mathbf{H}, \mathbf{D}, \boldsymbol{\Omega}, \mathbf{R}$ from the Eq. (13) to (15) as

\footnotetext{
${ }^{2}$ See Kim and Nelson (1999, pp. 100-103).

${ }^{3}$ See Kim and Nelson (1999, pp. 104-105) for details.

${ }^{4}$ All technical details concerning our estimation procedure and smoothing algorithm are available upon request.
} 
regime-switching parameters and collect them in the (two-state) switching matrices $\mathbf{F}_{S_{t}}, \mathbf{H}_{S_{t}}, \mathbf{D}_{S_{t}}, \boldsymbol{\Omega}_{S_{t}}, \mathbf{R}_{S_{t}}$. However, since it proves difficult to numerically maximize the $\log$ likelihood function of such a fully-fledged and highly-parameterized state-space model with Markov-switching, we reduce model complexity considerably by imposing the following restrictions on the model parameters. First, we model the dividend process $\left\{d_{t}\right\}$ as a pure random walk without drift, that is we set $\mu=0$ and $h=0$ in Eq. (6) thereby contracting the matrix D from Eq. (14) correspondingly. Second, we only allow the autoregressive coefficient $1 / \psi$ in the bubble process (10) to switch between the two regimes (in order to discriminate between moderately growing and explosive periods in the bubble process) while modeling all other parameters as non-switching between the Markov-regimes. Both restrictions imply that we ultimately estimate the switching autoregressive bubble coefficients $1 / \psi_{1}$ and $1 / \psi_{2}$ in the matrices $\mathbf{F}_{S_{t}}$, the non-switching variances $\sigma_{\eta}^{2}$ and $\sigma_{\delta}^{2}$ in the matrices $\Omega$ and $\mathbf{R}$ from Eq. (15) and both transition probabilities $p_{11}$ and $p_{22}$ from the matrix $\boldsymbol{\Pi}$ in Eq. (20).

\section{Empirical analysis}

In this section, we apply our two-regime Markov-switching state-space model to a plethora of bubble processes. Our analysis covers artificial as well as real-world data sets. We specify our artificial bubble processes in the sense of Evans (1991). Our real-world data consist of the well-known data set by Robert Shiller for the US plus a variety of Datastream price and dividend data for various countries that are known to have suffered from severe bubble episodes in the past (see Kindleberger and Aliber, 2005).

\subsection{Artificial and real-world datasets}

First, we address our artificial bubble processes. Evans (1991) describes an empirically plausible class of bubbles which are rational, positive and periodically collapsing. These bubbles have the form

$$
B_{t+1}=\left\{\begin{array}{rl}
(1+r) B_{t} u_{t+1}, & \text { if } B_{t} \leq \alpha \\
{\left[\delta+\frac{1+r}{\pi}\left(B_{t}-\frac{\delta}{1+r}\right) \xi_{t+1}\right] u_{t+1},} & \text { if } B_{t}>\alpha
\end{array},\right.
$$

where $\delta$ and $\alpha$ are real scalars such that $0<\delta<(1+r) \alpha$. $\left\{u_{t}\right\}$ is a sequence of non-negative exogenous i.i.d. random variables with $E_{t}\left(u_{t+1}\right)=1$, while $\left\{\xi_{t}\right\}$ is an exogenous i.i.d. Bernoulli process independent of $\left\{u_{t}\right\}$ with $\operatorname{Pr}\left(\xi_{t}=0\right)=1-\pi$ and 
$\operatorname{Pr}\left(\xi_{t}=1\right)=\pi$ for $0<\pi \leq 1$. This bubble process has two different rates of growth. For $B_{t} \leq \alpha$ the bubble grows at the mean rate $1+r$. For $B_{t}>\alpha$ it grows at the faster mean rate $(1+r) / \pi$, but collapses with probability $1-\pi$ per period. When the bubble collapses, it falls back to the mean value $\delta$ and the process recommences.

More explicitly, we assume the variables $\left\{u_{t}\right\}$ to be i.i.d. lognormally distributed and scaled to have unit mean, i.e. we assume $u_{t}=\exp \left(y_{t}-\tau^{2} / 2\right)$ with $\left\{y_{t}\right\}$ being i.i.d. $N\left(0, \tau^{2}\right)$. Altering the parameters $\delta, \alpha$ and $\pi$, we can modify (1) the frequency with which the bubble erupts, (2) the average length of time before the bubble collapses, and (3) the scaling of the bubble. For example, a high value of $\alpha$ generates bubbles with a long initial period of relatively steady but slow growth.

In order to apply our Markov-switching state-space approach from Section 4, we have to generate artificial dividend and stock-price data which we regard as fundamental stock-price data and on which we then superimpose Evans-bubbles of the form (29). ${ }^{5}$ For this, we assume that the data-generating process for the dividends follows a pure random walk,

$$
D_{t}=D_{t-1}+\epsilon_{t}
$$

where $\left\{\epsilon_{t}\right\}$ is a Gaussian white-noise process with mean zero and variance $\sigma_{\epsilon}^{2}$. Using the dividend DGP (30), we can derive the fundamental stock-price from the linear present-value relation (with constant expected returns) as

$$
P_{D_{t}}=r^{-1} D_{t}
$$

Now, we generate a bubbly stock-price process by adding the Evans-bubble (29) to the fundamental stock-price process $P_{D_{t}}$ from Eq. (31):

$$
P_{t}=P_{D_{t}}+B_{t}
$$

[Insert Table 1 here]

In Table 1 we have specified three parameterizations for the bubbly stock-price process according to the Eqs. (29) to (32). With each parameter specification, we have generated three distinct sets of $\left\{B_{t}\right\}^{-},\left\{D_{t}\right\}-,\left\{P_{D_{t}}\right\}^{-}$and $\left\{P_{t}\right\}$-trajectories. We denote the three sets of trajectories generated by the first specification given in column 1 of Table 1 by DGP 1a, DGP 1b, DGP 1c, and similarly the sets of trajectories for the parameter specifications given in the columns 2 and 3. In all specifications the bub-

\footnotetext{
${ }^{5}$ For a discussion as to what extent dividends may be viewed as appropriate stock-price fundamentals see, among others, Lamont (1998).
} 
ble and dividend parameters were chosen in line with the study of West (1988) who estimated these parameters on the basis of S\&P500 data ranging between 1871 and 1980.

Next, we address our real-world data sets. We analyze data for the US, Brazil, Indonesia, Malaysia and Japan. Except for the US data set, which is provided by Robert Shiller ${ }^{6}$, all other data were compiled from Datastream. All data are provided on a monthly basis.

The respective time spans differ among the countries under consideration. The largest time span is available for the US with monthly data ranging between January 1871 and June 2004 which amounts to a total number of 1602 observations. In our empirical analysis, we use four variants of the US data, namely (1) the complete data set, (2) a first subsample covering data between January 1871 and December 1912 (504 observations), (3) a second subsample covering data between January 1913 and December 1954 (504 observations), and (4) a final subsample including data between January 1955 and June 2004 (594 observations). The reason for this splitting up the whole US sample is that it turns out to be easier to trace historical events when working with the three subsamples.

The respective sampling periods for Brazil, Indonesia, Malaysia and Japan are August 1994 until October 2005 (135 observations), April 1990 until October 2005 (187 observations), February 1986 until October 2005 (237 observations) and January 1973 until October 2005 (394 observations).

\section{[Insert Tables 2 to 5 here]}

\subsection{Estimation results}

We begin with formal specification testing of the Markov-switching model against linear alternatives. Hansen $(1992,1996)$ and Garcia (1998) propose a standardized likelihood ratio (LR) test in order to provide (asymptotically) valid inference. Hansen's (1992) approach gives a bound on the asymptotic null-distribution of the standardized LR test. However, this test procedure is computationally demanding and infeasible in our state-space estimation framework. By contrast, in a predecessor version of Ang and Bekaert (2002), the authors suggest that the true underlying null-distribution of the conventional LR test can be approximated by a $\chi^{2}(q)$ distribution, where the degreeof-freedom parameter $q$ equals the number of linearly independent restrictions under the null hypothesis. Table 2 displays the LR test statistics for the artificial Evans-

\footnotetext{
${ }^{6}$ Cf. the website http://www.econ.yale.edu/shiller/.
} 
processes. The LR test statistics are so extreme that they exceed all critical values used in practice, thus endorsing the Markov-switching specification for our artifical data sets. Even more overwhelming evidence in favor of Markov-switching is found for all our real-world data sets in Table 4.

Next, we analyze the point estimates of our state-space models with Markov-switching. We first consider the trajectories of the Evans-processes and then address our real-world data sets. Table 3 reveals that the bubble parameters $1 / \psi_{1}$ and $1 / \psi_{2}$ are significantly different from zero for the majority of the Evans-processes. Moreover, it is obvious without formal statistical testing that the majority of the estimated bubble coefficients are significantly different from each other across both regimes. The standard deviations $\sigma_{\eta}$ of the bubble innovation term are significantly different from zero at the $5 \%$ level. By contrast, the results for the standard deviation $\sigma_{\delta}$ of the dividend innovation term appear to be less clear-cut. For the DGPs 1a, 2b, 2c and 3a we cannot reject the null hypothesis ' $\sigma_{\delta}=0$ '. All estimates of the transition probabilities $p_{11}$ and $p_{22}$, although not statistically significant at conventional significance levels, are within the expected range.

Table 5 displays the estimation result for our real-world data sets. Here, the bubble parameters $1 / \psi_{1}$ and $1 / \psi_{2}$ are significant at conventional levels for all our samples. Also, most of the bubble-parameters appear to differ significantly from each other across both regimes. The standard deviations $\sigma_{\eta}$ of the bubble innovation are all insignificant while, by contrast, all standard deviations $\sigma_{\delta}$ of the dividend processes are significantly different from zero at conventional significance levels. Again, the transition probabilities $p_{11}$ and $p_{22}$ are within the expected ranges.

[Insert Figures 1 to 3 here]

Figures 1 to 3 display the smoothed regime-1 probabilities along with the respective fundamental and price processes for the nine artificial Evans data sets. The two upper panels in Figure 1 refer to the Evans-process 1a for which our method is capable of indentifying three out of four bubbles. Only the second bubble, which lies in the first half of the sample, is not detected. For the Evans-process $1 \mathrm{~b}$ three bubbles exist two of which are correctly identified by our methodology. Here, the unidentified bubble is located in the second half of the sample. Finally, for the Evans-process 1c all bubbles are correctly detected. In contrast to the Evans-processes 1a and 1b, the four bubbles in the Evans-process 1c are of rather equal size. From this, one may conjecture that our methodology is more successful in identifying homogeneously sized bubbles than 
heterogeneously sized bubbles.

For the Evans-process 2a (Figure 2) we see that the first huge price bubble is followed by two tiny bubbles right after the crash of the initial huge bubble (occuring at the observations 30 and 33). Here, our methodolgy detects the huge bubble but misses both tiny bubbles. As time elapses, two further moderate bubbles arise both of which are identified. We conjecture that our procedure has difficulties in detecting bubbles that emerge immediately after the collapse of a preceding bubble.

In the Evans-process $2 \mathrm{~b}$ we have a huge price bubble at the end of the sample which is preceded by two small and one moderately sized bubbles. All four bubbles are detected. Finally, the Evans-process 2c produces five bubbles. In this case, our method only identifies the largest bubble in the sample.

Figure 3 displays the Evans-processes 3a, 3b and 3c. Here, the main difference to the former Evans-trajectories is that the sample length was doubled to 200 observations. As a result, the number of bubbles is considerably higher. For the Evans-process 3 a only two collapsing bubbles are detected, the first located at the beginning, the second located at the end of the sample. Obviously, our technology fails in identifying intermediate bubbles. A similar result obtains for the Evans-process $3 \mathrm{~b}$ where two collapsing bubbles at the end of the sample can be identified. The Evans-process 3c produces more collapsing bubbles than the Evans-processes 3a and 3b, many of which are identified by our technology (particularly in the first half of the sample).

\section{[Insert Figures 4 to 6 here]}

Finally, we analyze our real-world data sets. The Figures 4 to 6 display the smoothed regime-1 probabilities along with the price indexes for the respective countries. Figure 4 contains the results for the entire US data set ranging from 1871 to 2004. Here, the smoothed probabilities clearly reflect the start of the great depression in 1929 and the turmoil in world-wide financial markets prior to the beginning of the second World War. However, since the complete time span analyzed covers more than 130 years, we have split up the whole sample into three subsamples covering the periods 1871-1912, 1913-1954 and 1954-2004, respectively.

For the first US subsample (middle panels in Figure 4) we observe eruptions in the smoothed regime-1 probabilities in the years 1873, 1877, 1880, 1886, 1893, 1900, 1904 and 1907. Except for the year 1900, the eruptions appear to be short-lived and rather indicate minor crashes than longer-lasting speculative bubbles or collapsing periods. For the second US subsample (lower panels in Figure 4) the number of eruptions in the 
smoothed probabilities is considerably smaller than in the first subsample. However, our technology correctly indicates the peak of the 1929-bubble and the turmoil in American financial markets prior to World War II.

In the third US subsample (upper panels in Figure 5) we observe strong eruptions in the smoothed probabilities in the years 1963, 1973, 1977, 1987 until 1990 as well as at the end of the 2002. The eruptions in 1973, 1987 and 2002 respectively reflect the first oil crisis, the black monday and the collapse of the internet bubble. There is another less pronounced peak in the smoothed probabilities in 1979 (not shaded in Figure 5) reflecting the second oil crisis. The other more pronounced eruptions (like those in 1963) might reflect minor crises that so far have not been described in the bubble literature.

The middle panels in Figure 5 display the results for Brazil between 1994 and 2005. The smoothed probabilities exhibit eruptions during 1998 as well as at the end/beginning of 1999/2000. The eruptions in 1998 can be explained by the repercussion of the East Asian crisis, while the peak in 1999/2000 reflects the Brazilian currency crisis.

In the smoothed regime-1 probabilities for Indonesia (lower panels of Figure 5) we find three periods characterized by strong eruptions. The first lasts from the end of 1990 until mid-1992, the second from 1998 until the beginning of 2000, while the third ranges from the end of 2001 until 2004. While we cannot explain the first wave of eruptions from historical facts, we respectively attribute the second and the third wave to the East Asian crisis and the collapse dot-com bubble with their long-lasting impacts on many financial markets world-wide.

The last countries to be discussed are Malaysia and Japan (see Figure 6). For Malaysia, we observe three periods with strong eruptions, namely (1) from the end of 1986 until the beginning of 1988, (2) from the beginning of 1994 until the end of 1994, and (3) from the end of 1997 until the end of 2000. Here again, the third wave reflects the East Asian crisis. The smoothed probabilities for Japan unambiguously indicate the implosion of the famous Japanese bubble around the beginning of the year 1990. It is interesting to note, however, that Kindleberger and Aliber (2005) report three other bubbles in Japan between 1985 and 2000 which our technology does not detect.

\section{Concluding remarks}

In this paper we propose a new methodology for detecting speculative bubbles in stockprice data. The technology constitutes a state-space approach that is enriched by 
Markov-switching elements. Up to now, this procedure has mainly been used in the field of business-cycle research. Our innovation is to adapt this technology to Campbell and Shiller's (1988a, b) present-value stock-price model. In the state-space representation of this model, the unobservable state vector constitutes the bubble component and by allowing the bubble process to switch between two regimes according to a firstorder Markov chain, we are able to statistically discriminate moderately growing from explosive periods in the bubble process.

In order to check the validity of our econometric procedure, we apply it to a number artificial bubble processes which we generate according to the algorithm suggested by Evans (1991). Furthermore, we analyze real-world data using the well-known stock and dividend data set by Robert Shiller as well as stock-price data for various other countries which are known to have experienced severe bubble periods.

The results of our investigation are twofold. First, we find statistically significant regime-switching structures in the stock-price bubble processes of our real-world data sets. Consequently, regime-switching should be taken into account in any theoretical bubble-model and, in particular, should be included in econometric specifications. Second, our Markov-switching approach is able to detect many bubbles in our artificial Evans-processes as well as in our real-world data sets. For the countries considered, our (smoothed) regime-probability technique identifies most speculative periods as classified by Kindleberger and Aliber (1995).

A potential line of future research might start from our empirical observation that Markov-switching specifications may adequately capture the dynamics of stock-price bubbles. This knowledge should be integrated into conventional methods designed to detect real-time stock-price bubbles. Furthermore, it would be interesting to apply our procedure to other artificial bubble processes, for example to those suggested by Charemza and Deadman (1995).

\section{References}

Abreu, D., Brunnermeier, M. (2003). Bubbles and Crashes. Econometrica 71, 173-204. Allen, F., Gale, D. (2000). Bubbles and Crises. Economic Journal 110, 236-255.

Allen, F., Morris, S., Postlewaite, A. (1993). Finite Bubble With Short Sale Constraints and Asymmetric Information. Journal of Economic Theory 61, 206-229.

Ang, A., Bekaert, G. (2002). Regime Switches in Interest Rates. Journal of Business \& Economic Statistics 20(2), 163-182.

Brooks, C., Katsaris, A. (2005). A Three-Regime Model of Speculative Behaviour: Modelling the Evolution of the S\&P 500 Composite Index. The Economic Journal 
$115,767-797$.

Campbell, J.Y., Shiller, R.J. (1987). Cointegration and Tests of Present Value Models. Journal of Political Economy 95(5), 1062-1088.

Campbell, J.Y., Shiller, R.J. (1988a). The Dividend-Price Ratio and the Expectations of Future Dividends and Discount Factors. Review of Financial Studies 1(3), 195-228.

Campbell, J.Y., Shiller, R.J. (1988b). Stock Prices, Earnings and Expected Dividends. Journal of Finance 43(3), 661-676.

Charemza, W., Deadman, D. (1995). Speculative Bubbles with Stochastic Explosive Roots: the Failure of Unit Root. Journal of Empirical Finance 2, 153-163.

Chauvet, M. (1998). An Econometric Characterization of Business Cycle Dynamics with Factor Structure and Regime Switching. International Economic Review 39, 969-996.

Chauvet, M., Hamilton, J.D. (2006). Dating Business Cycle Turning Points. In: Nonlinear Time Series Analysis of Business Cycles. Milas, C., Rothman, P., van Dijk, D. (Eds), Elsevier B.V., Amsterdam, pp. 1-54.

Chauvet, M., Piger, J.M. (2003). Identifying Business Cycle Turning Points in Real Time. Federal Reserve Bank of Saint Louis Review, March/April, 47-62.

Cuthbertson, K., Nitzsche, D. (2004). Quantitative Financial Economics: Stocks, Bonds and Foreign Exchange. 2nd Edition, Wiley, New York.

Diba, B.T., Grossman, H.I. (1988). Explosive Rational Bubbles in Stock Prices? American Economic Review 78, 520-530.

Driffill, J., Sola, M. (1998). Intrinsic Bubbles and Regime-Switching. Journal of Monetary Economics 42, 357-373.

Evans, G.W. (1991). Pitfalls in Testing for Explosive Bubbles in Asset Prices. American Economic Review 81, 922-930.

Garcia, R. (1998). Asymptotic Null Distribution of the Likelihood Ratio Test in Markov Switching Models. International Economic Review 39, 763-788.

Gordon, K., Smith, A.F.M. (1988). Modeling and Monitoring Discontinuous Changes in Time Series. In: Bayesian Analysis of Time Series and Dynamic Linear Models. Spall, J. (Ed), Marcel Dekker, New York, pp. 359-392.

Hall, S.G., Psaradakis, Z., Sola, M. (1999). Detecting Periodically Collapsing Bubbles: A Markov-Switching Unit Root Test. Journal of Applied Econometrics 14, 143154.

Hamilton, J.D. (1988). Rational-Expectations Econometric Analysis of Changes in Regime: An Investigation of the Term Structure of Interest Rates. Journal of Economic Dynamics and Control 12, 385-423. 
Hamilton, J.D. (1989). A New Approach to the Economic Analysis of Nonstationary Time Series and the Business Cycle. Econometrica 57, 357-384.

Hamilton, J.D. (1994a). Time Series Analysis. Princeton University Press, Princeton, New Jersey.

Hamilton, J.D. (1994b). State-Space Models. In: Handbook of Econometrics. Engle, R.F., McFadden, D. (Eds). Elsevier, pp. 3039-3080.

Hansen, B.E. (1992). The Likelihood Ratio Test under Nonstandard Conditions: Testing the Markov Switching Model of GNP. Journal of Applied Econometrics 7(S), S61-S82.

Hansen, B.E. (1996). Erratum: The Likelihood Ratio Test under Nonstandard Conditions: Testing the Markov Switching Model of GNP. Journal of Applied Econometrics 11(2), 195-198.

Harrison, P.J., Stevens, C. (1976). Bayesian Forecasting. Journal of the Royal Statistical Society, B38, 205-247.

Kim, C.-J., Nelson, C.R. (1999). State Space Models with Regime Switching. MIT Press, Cambridge.

Kindleberger, C.P., Aliber, R.Z. (2005). Maniacs, Panics, and Crashes. A History of Financial Crises. 5th Edition, John Wiley and Sons, Hoboken, New Jersey.

Kitagawa, G. (1987). Non-Gaussian State-Space Modeling of Nonstationary Time Series. Journal of American Statistical Association 82, Theory and Method, 1032-1063.

Lamont, O. (1998). Earnings and Expected Returns. Journal of Finance 53, 1563-1587.

McMillan, D.G. (2007). Bubbles in the Dividend-Price Ratio? Evidence From an Asymmetric Exponential Smooth-Transition Model. Journal of Banking and Finance $31,787-804$.

Smith, A.F.M., Makov, U.E. (1980). Baysian Detection and Estimation of Jumps in Linear Systems. In: Analysis and Optimization of Stochastic Systems. Jacobs, O. et al. (Eds), Academic Press, New York, pp. 333-345.

Tirole, J. (1982). On the Possibility of Speculation Under Rational Expectations. Econometrica 50, 1163-1181.

Vigfusson, R., Van Norden, S. (1998). Avoiding the Pitfalls: Can Regime-Switching Tests Detect Bubbles? Studies in Nonlinear Dynamics and Econometrics 3, 1-22.

West,K.D. (1987). A Specification Test for Speculative Bubbles. Quarterly Journal of Economics 102, 553-580.

West, K.D. (1988). Dividend Innovations and Stock Price Volatility. Econometrica 56, $37-61$. 
Wu, Y. (1995). Are there rational bubbles in foreign exchange markets? Evidence from an alternative test. Journal of International Money and Finance 14, 27-46.

Wu, Y. (1997). Rational Bubbles in the Stock Market: Accounting for the U.S. StockPrice Volatility. Economic Inquiry 35, 309-319. 


\section{Figures and Tables}


Smoothed probabilities for Evans-process 1a

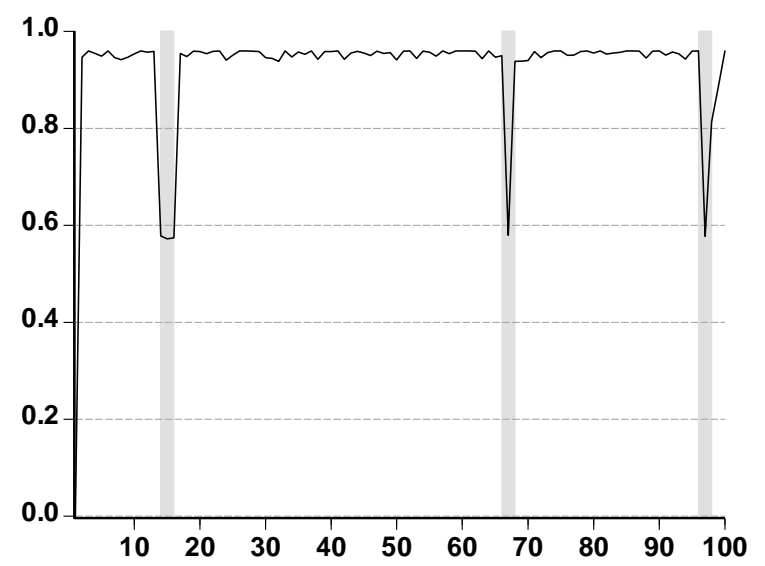

Smoothed probabilities for Evans-process 1b

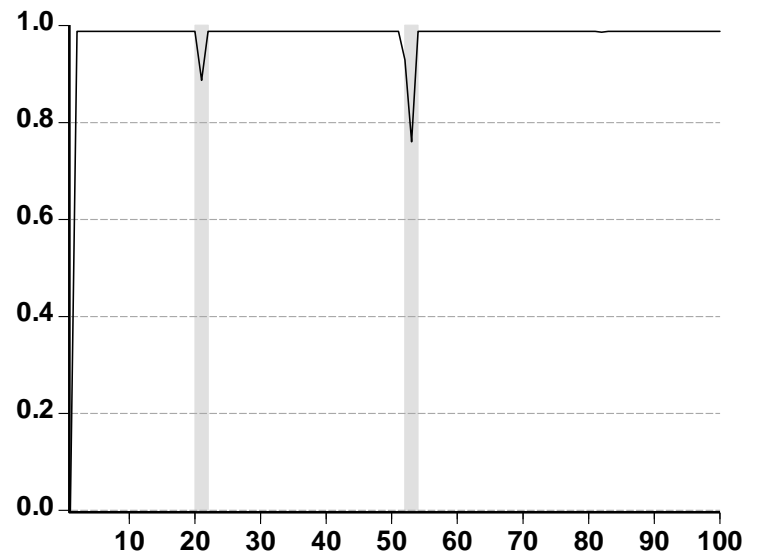

Smoothed probabilities for Evans-process 1c

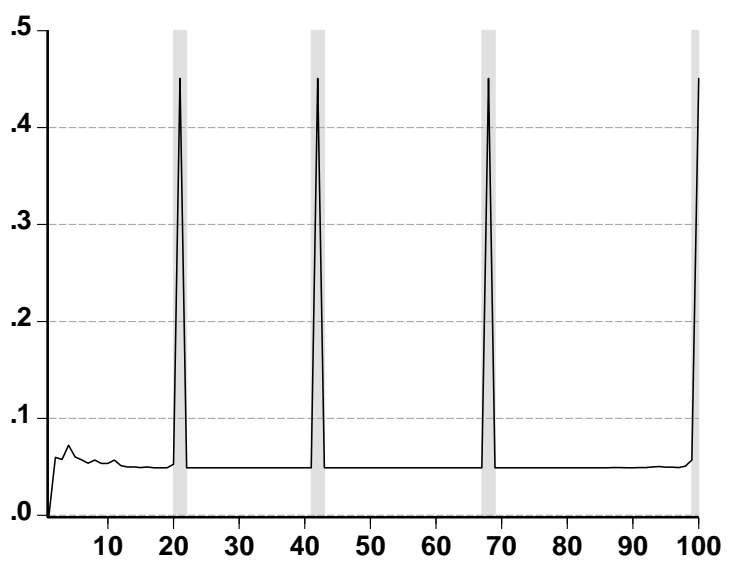

Price process for Evans-process 1a

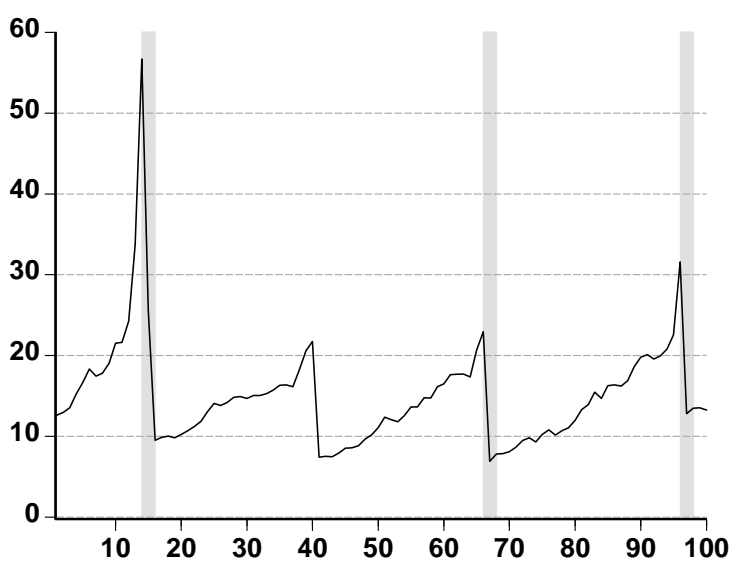

Price process for Evans-process 1b

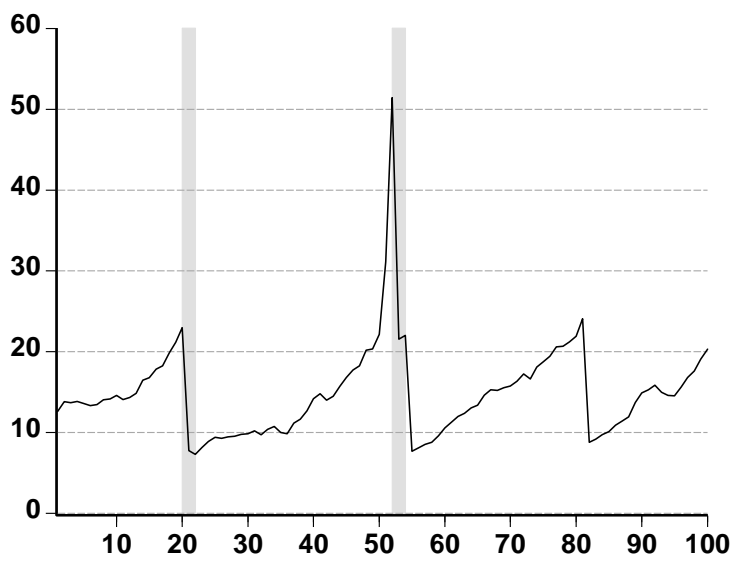

Price process for Evans-process 1c

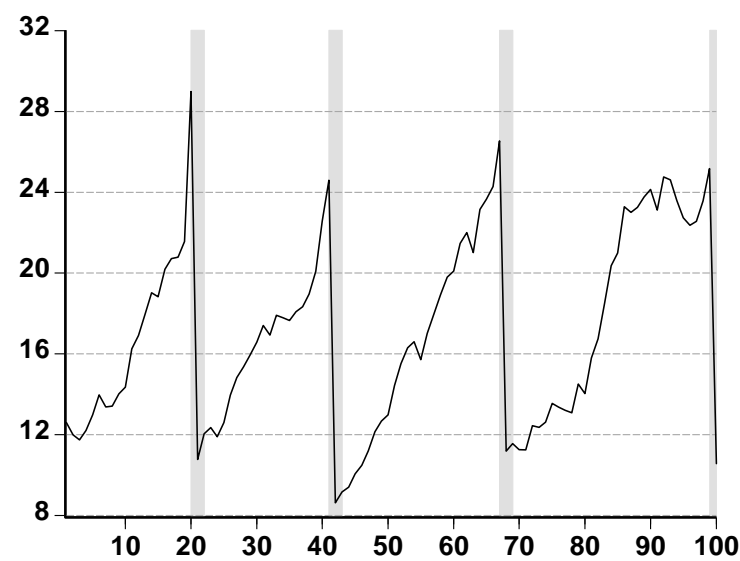

Figure 1: Smoothed regime-1 probabilities and price processes for the Evans-processes 1a, 1b, 1c 
Smoothed probabilities for Evans-process 2a

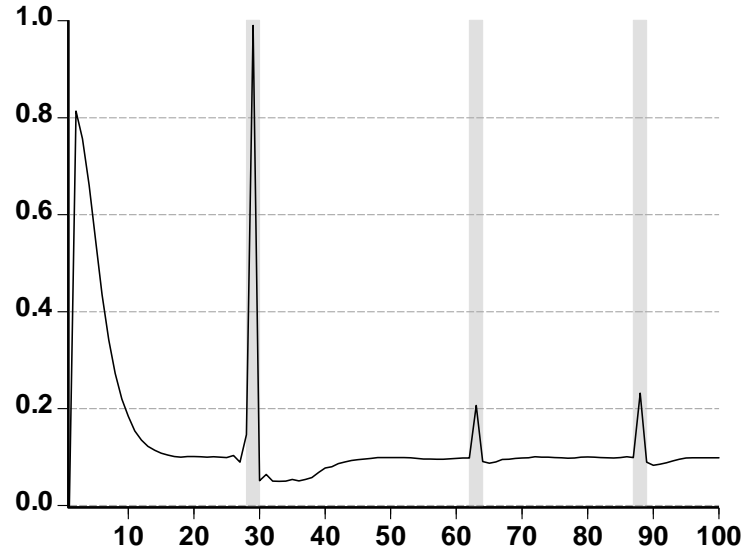

Smoothed probabilities for Evans-process $2 b$

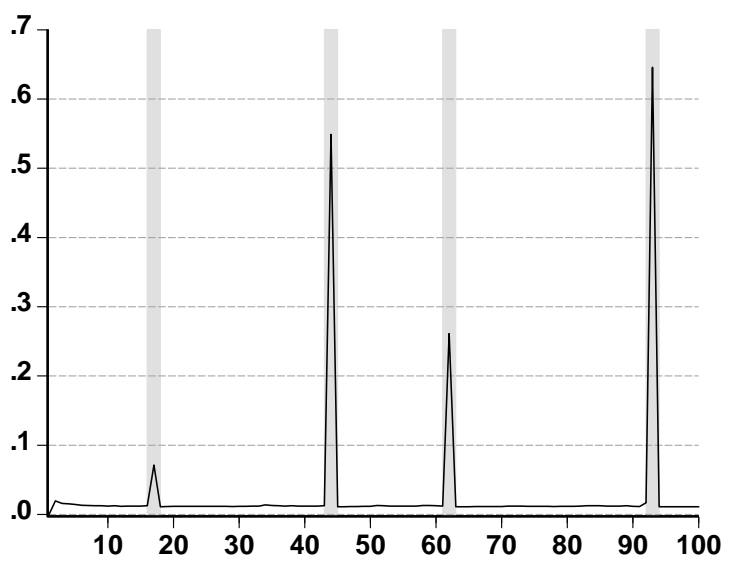

Smoothed probabilities for Evans-process 2c

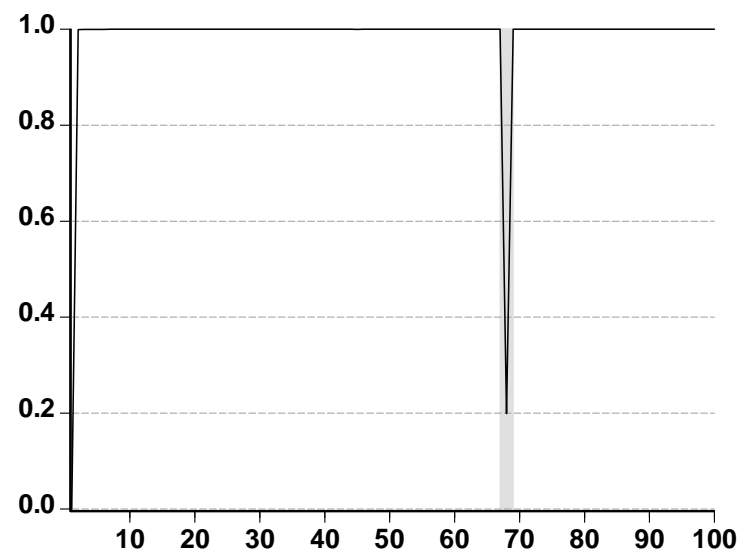

Price process for Evans-process 2a

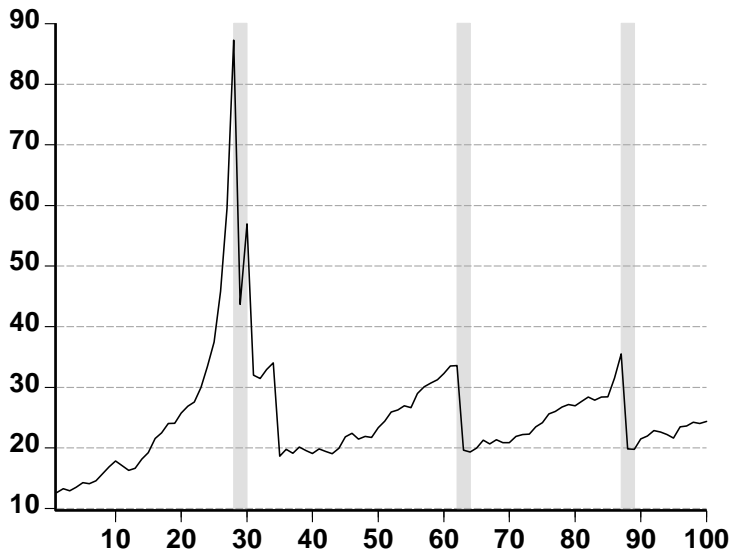

Price process for Evans-process $2 b$

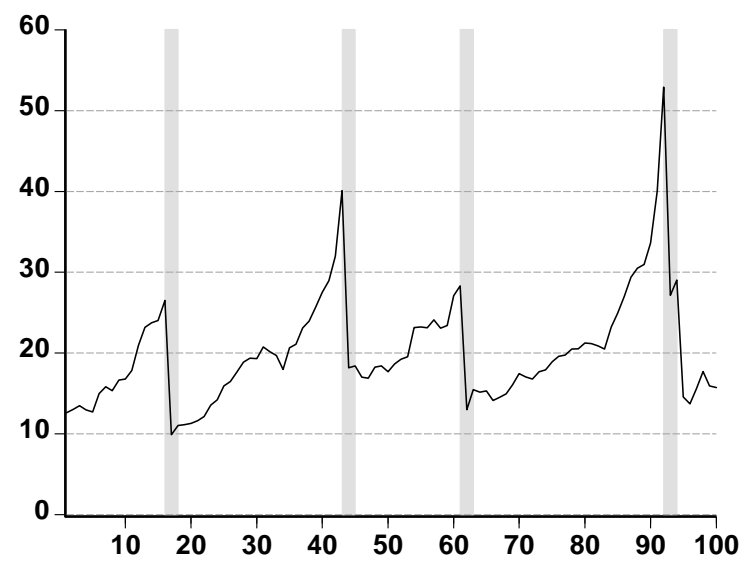

Price process for Evans-process 2c

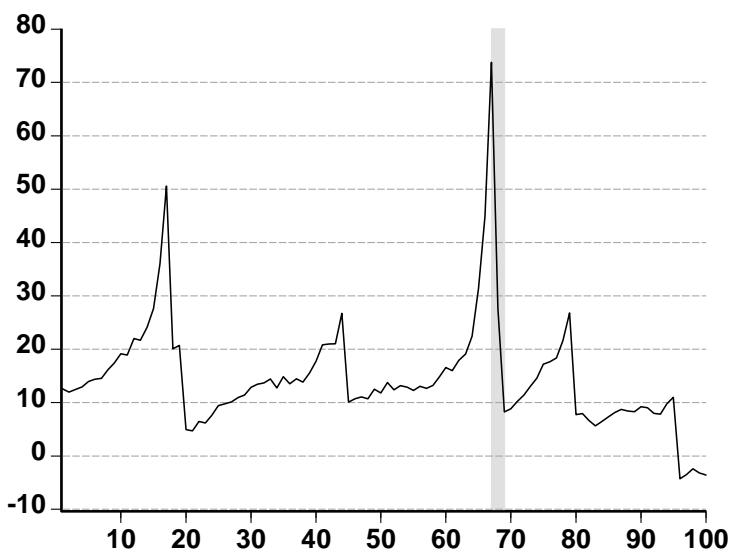

Figure 2: Smoothed regime-1 probabilities and price processes for the Evans-processes $2 \mathrm{a}, 2 \mathrm{~b}, 2 \mathrm{c}$ 
Smoothed probabilities for Evans-process 3a

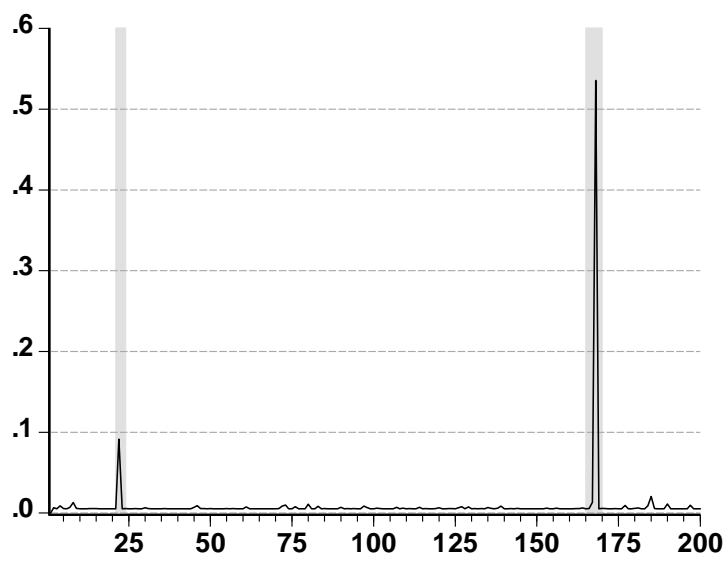

Smoothed probabilities for price process $3 b$

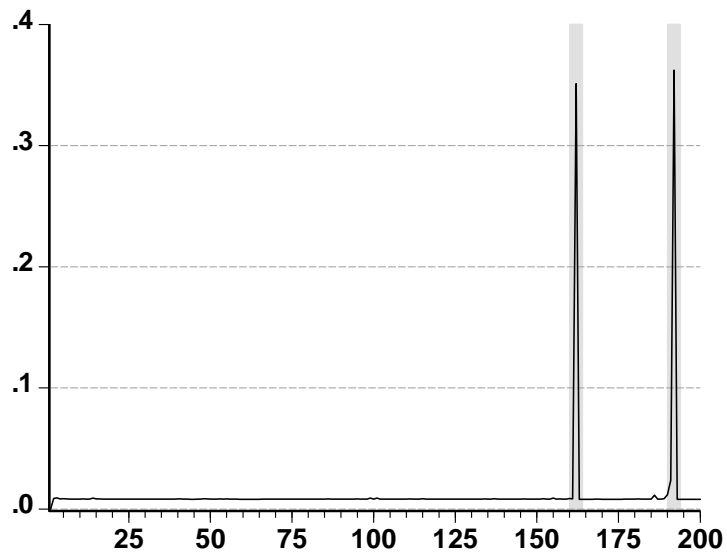

Smoothed probabilities for Evans-process 3c

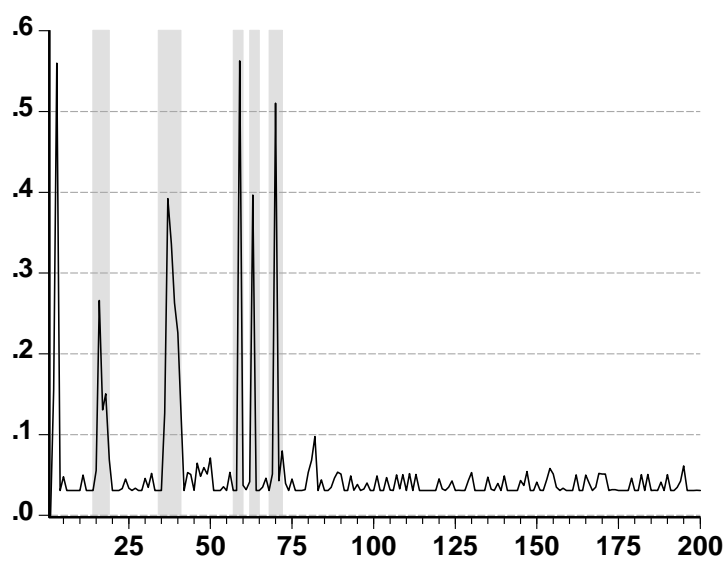

Price process for Evans-process 3a

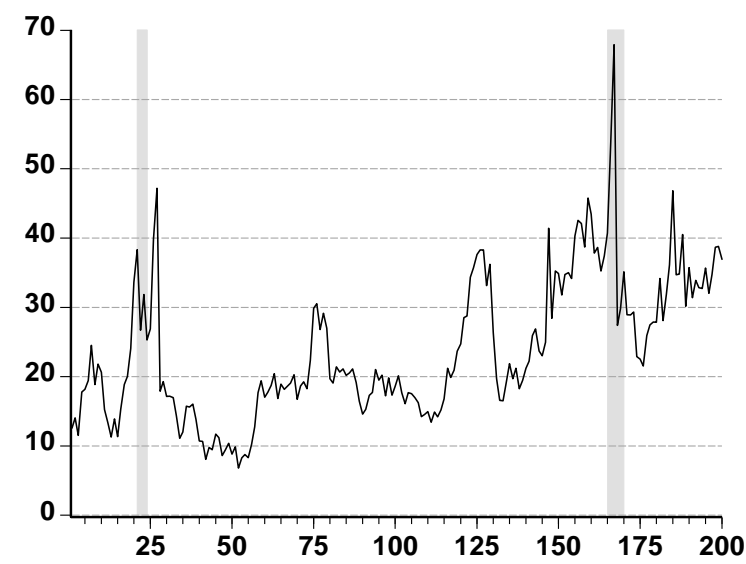

Price process for Evans-process 3b

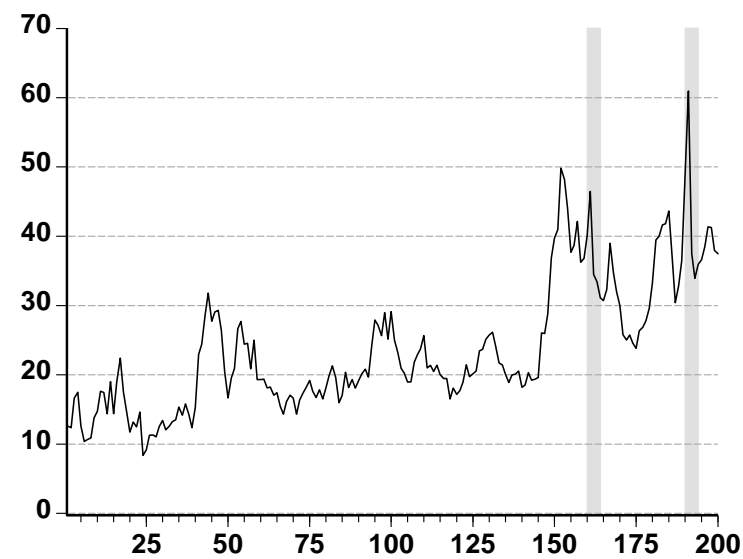

Price process for Evans-process 3c

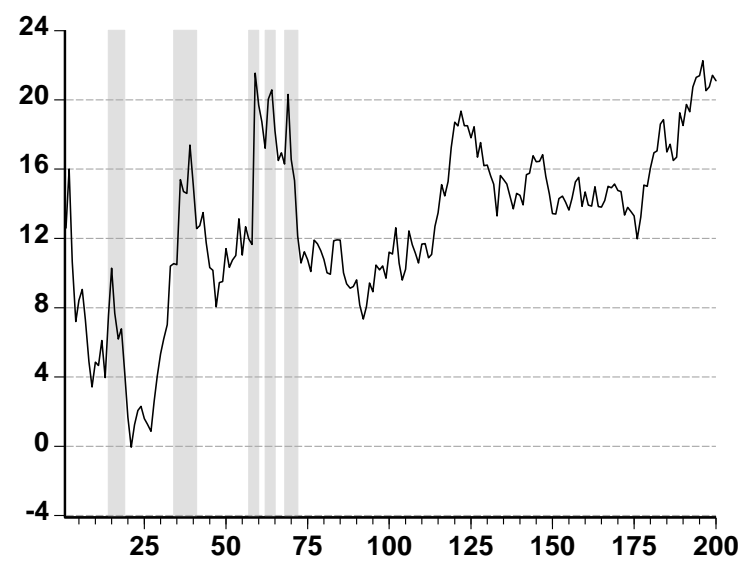

Figure 3: Smoothed regime-1 probabilities and price processes for the Evans-processes $3 \mathrm{a}, 3 \mathrm{~b}, 3 \mathrm{c}$ 
Smoothed probabilities, USA 1871-2004

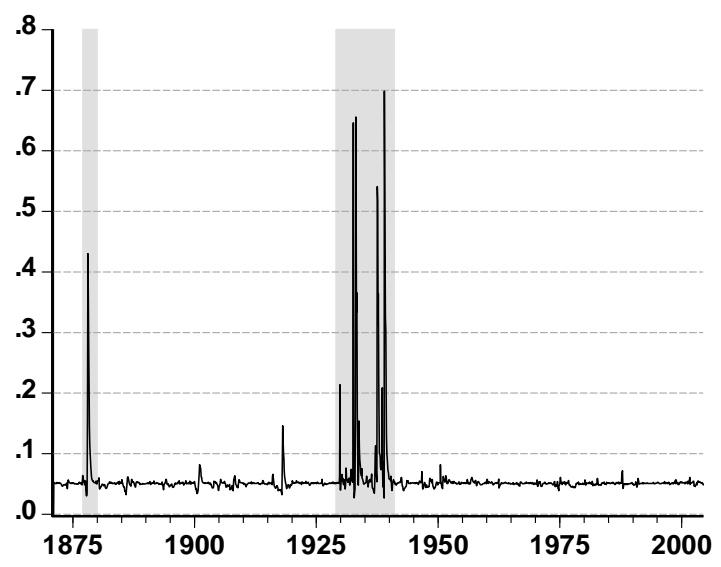

Smoothed probabilities, USA 1871-1912

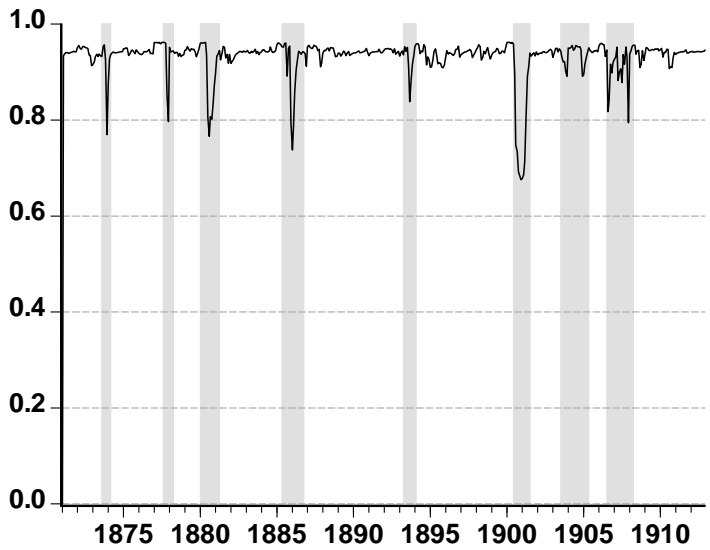

Smoothed probabilities, USA 1913-1954

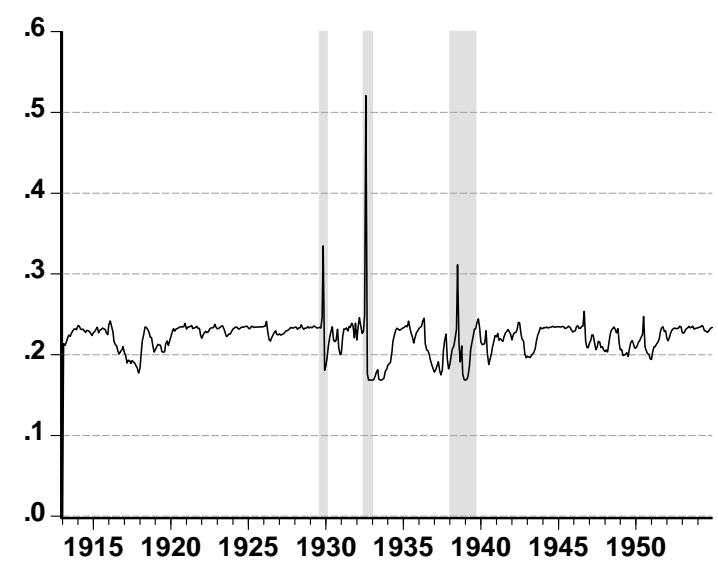

Prices (in logs), USA 1871-2004

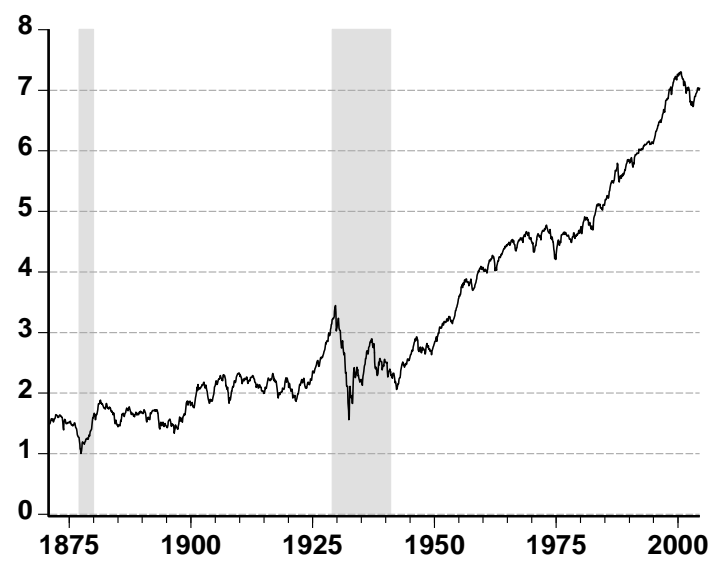

Prices (in logs), USA 1871-1912

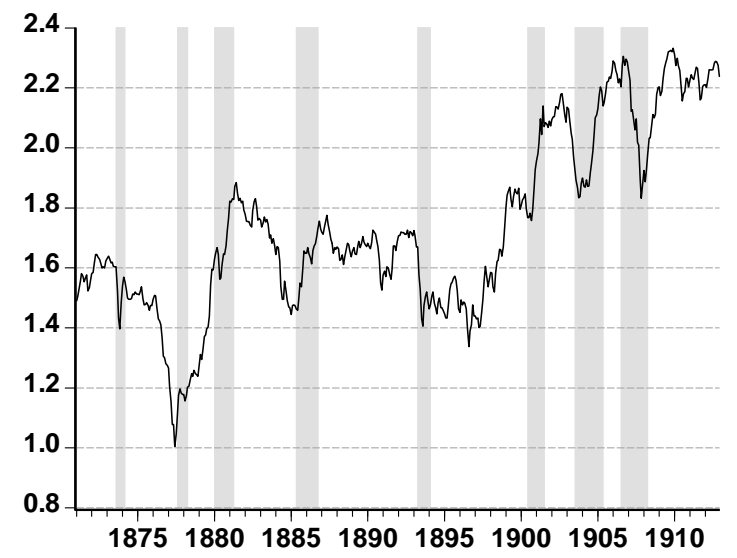

Prices (in logs), USA 1913-1954

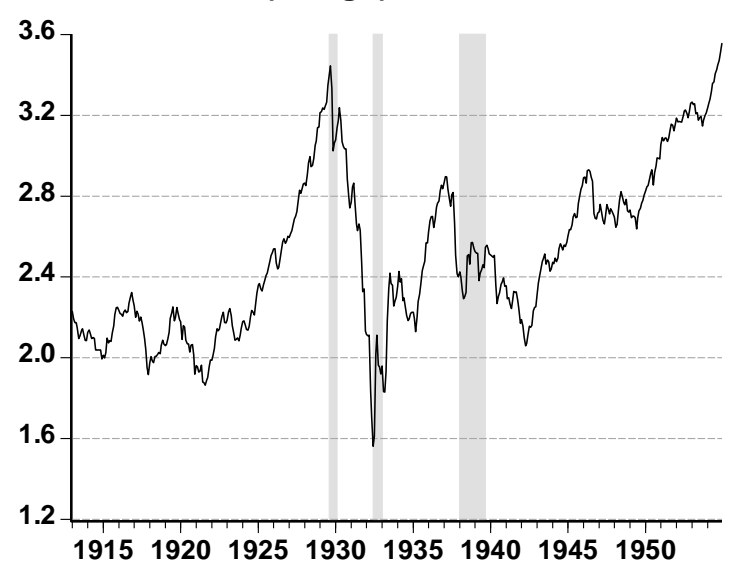

Figure 4: Smoothed regime-1 probabilities and stock prices for the USA (1871-2004, 1871-1912, 1913-1954) 
Smoothed probabilities, USA 1955-2004

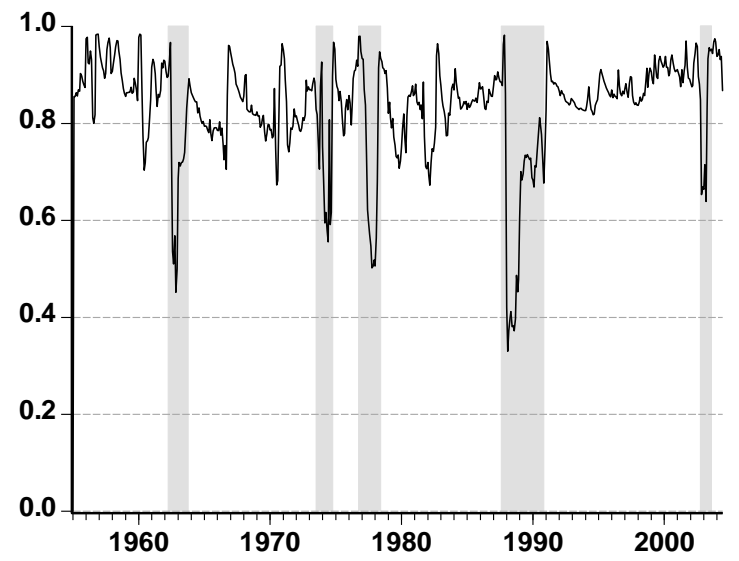

Smoothed probabilities, Brazil 1994-2005

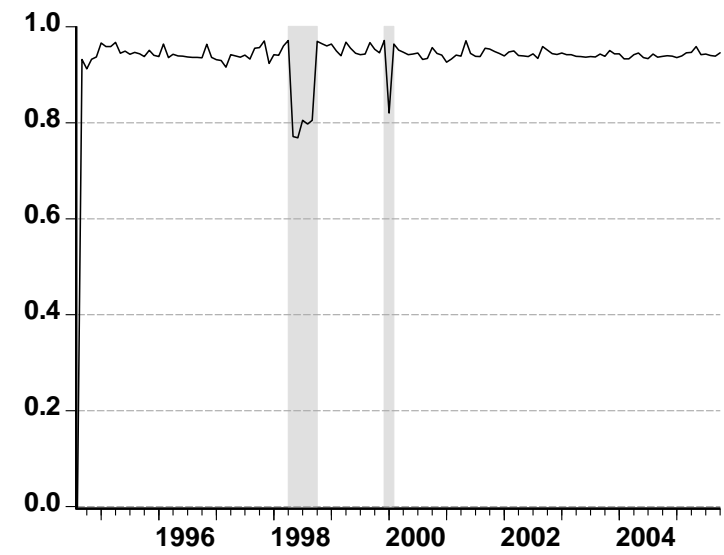

Smoothed probabilities, Indonesia 1990-2005

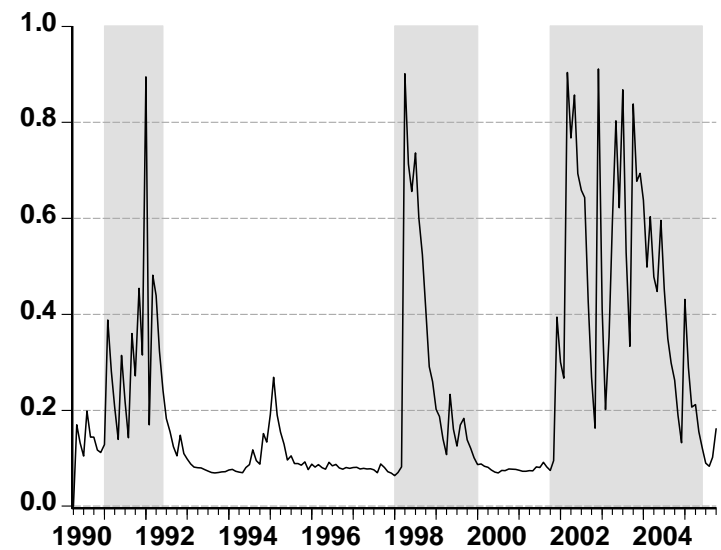

Prices (in logs), USA 1955-2004

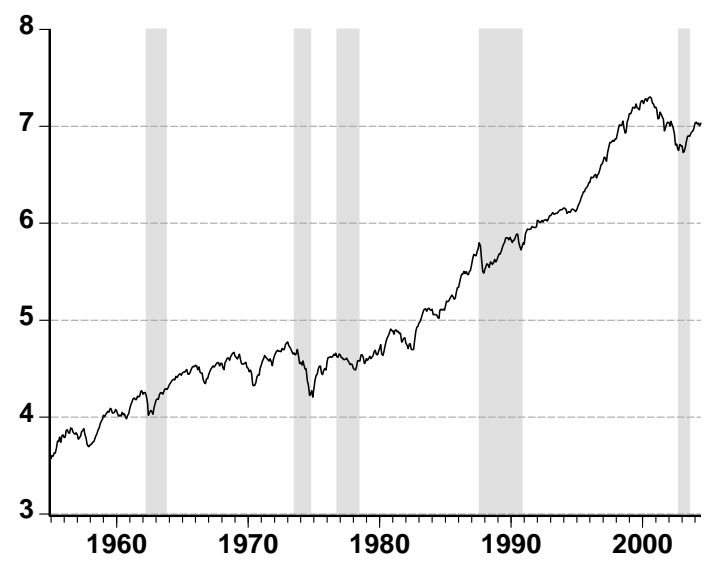

Prices (in logs), Brazil 1994-2005

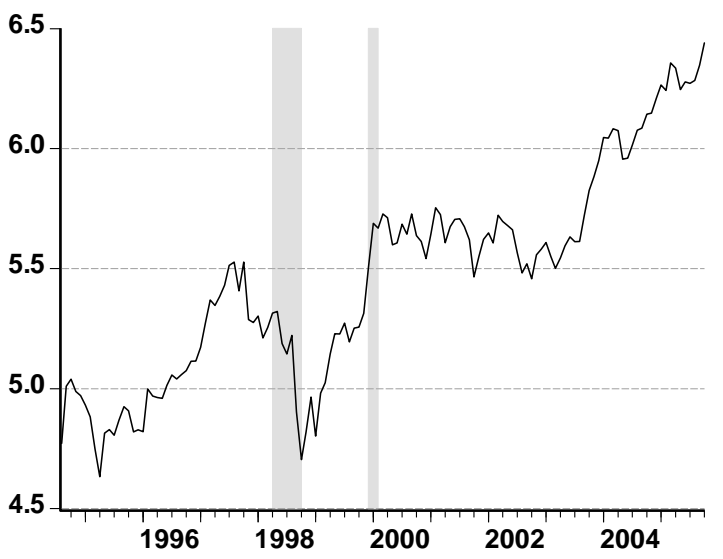

Prices (in logs), Indonesia 1990-2005

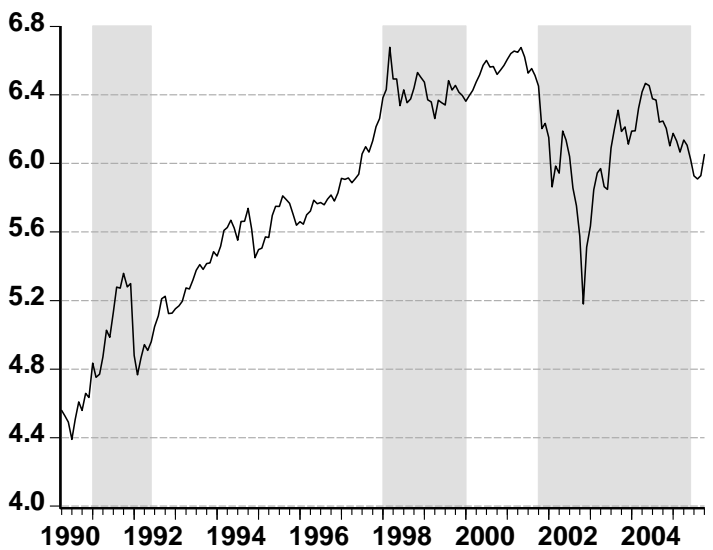

Figure 5: Smoothed regime-1 probabilities and stock prices for the USA (1955-2004), Brazil (1994-2005), Indonesia (1990-2005) 
Smoothed probabilities, Malaysia 1986-2005

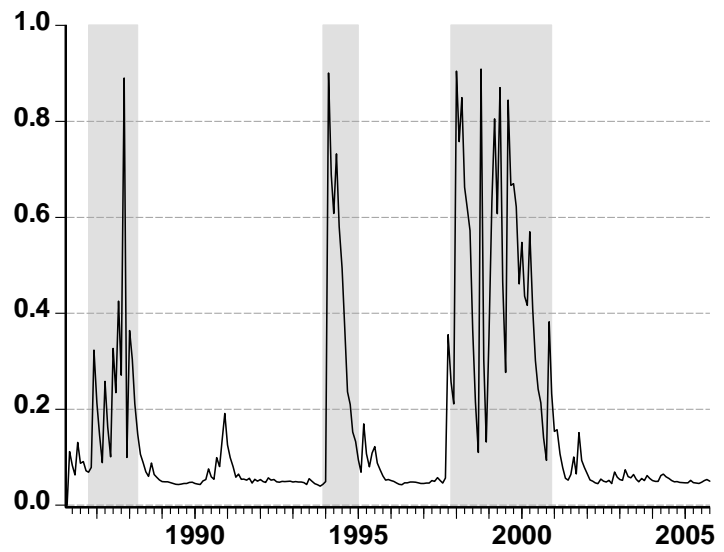

Smoothed probabilities, Japan 1973-2005

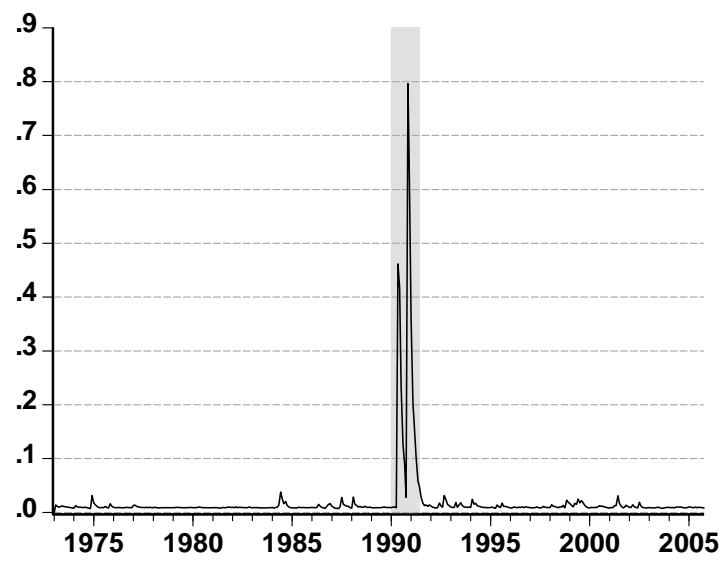

Prices (in logs), Malaysia 1986-2005

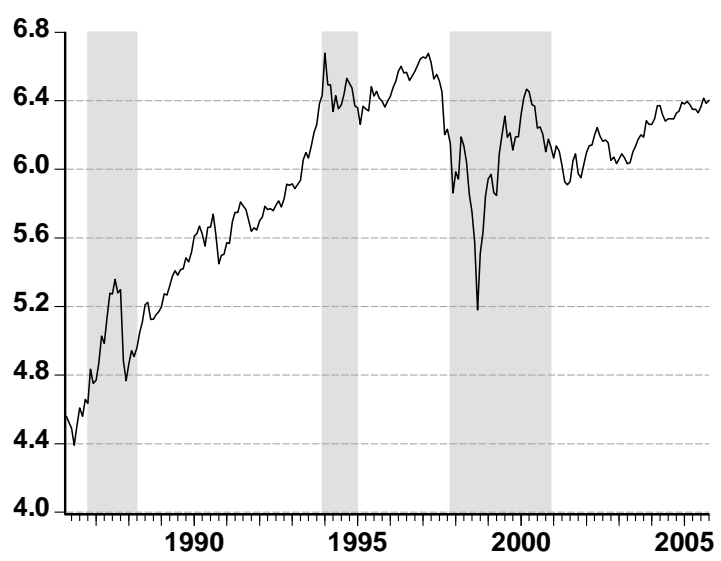

Prices (in logs), Japan 1973-2005

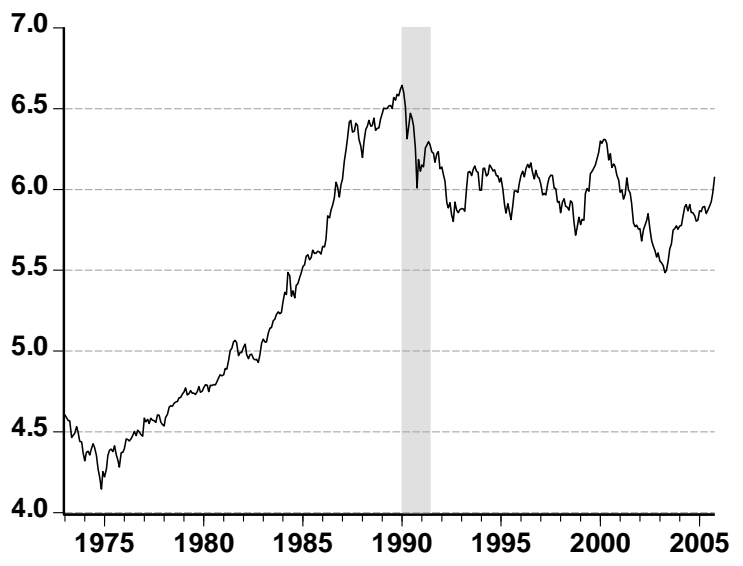

Figure 6: Smoothed regime-1 probabilities and stock prices for Malaysia (1986-2005), Japan (1973-2005) 
Table 1

Parameter specifications for the bubbly stock-price processes from Eqs. (29) to (32)

\begin{tabular}{llll}
\hline & \multicolumn{1}{c}{ DGP } & \multicolumn{1}{c}{ DGP } & \multicolumn{1}{c}{ DGP } \\
& $1 \mathrm{a}, 1 \mathrm{~b}, 1 \mathrm{c}$ & $2 \mathrm{a}, 2 \mathrm{~b}, 2 \mathrm{c}$ & $3 \mathrm{a}, 3 \mathrm{~b}, 3 \mathrm{c}$ \\
\hline Number of observations & 100 & 100 & 200 \\
$\alpha$ & 1.0000 & 1.0000 & 1.0000 \\
$\tau^{2}$ & 0.0025 & 0.0025 & 0.0025 \\
$r$ & 0.0500 & 0.0500 & 0.0500 \\
$\delta$ & 0.5000 & 0.5000 & 0.5000 \\
$D_{0}$ & 1.3000 & 1.3000 & 1.3000 \\
$B_{0}$ & 0.5000 & 0.5000 & 0.5000 \\
$\sigma_{\epsilon}^{2}$ & 0.1574 & 0.1574 & 0.1574 \\
$\pi$ & 0.8500 & 0.5000 & 0.5000 \\
Scaling of the bubble & 20.0000 & 20.0000 & 20.0000 \\
\hline
\end{tabular}


Table 2

Tests for Markov-switching property

\begin{tabular}{llll}
\hline & $\begin{array}{l}\text { Log Likelihood } \\
\text { Markov-switching } \\
\text { Model }\end{array}$ & $\begin{array}{l}\text { Log Likelihood } \\
\text { Linear } \\
\text { Model }\end{array}$ & $\begin{array}{l}\text { Likelihood Ratio } \\
\text { Test } \\
\text { Statistic }\end{array}$ \\
\hline DGP 1a & 292.8283 & 52.5920 & 480.4726 \\
DGP 1b & 295.2181 & 51.0532 & 488.3298 \\
DGP 1c & 369.2432 & 26.8172 & 684.8520 \\
DGP 2a & 370.7072 & 214.2220 & 312.9704 \\
DGP 2b & 311.9050 & 184.0089 & 255.7922 \\
DGP 2c & 357.5659 & 226.8750 & 261.3818 \\
DGP 3a & 618.9616 & 163.9522 & 910.0188 \\
DGP 3b & 552.5225 & 112.7132 & 879.6186 \\
DGP 3c & 368.1439 & 94.1706 & 547.9466 \\
\hline
\end{tabular}


Table 3

State-space model with Markov-switching

\begin{tabular}{cccccccc}
\hline & $1 / \psi_{1}$ & $1 / \psi_{2}$ & $\sigma_{\eta}$ & $\sigma_{\delta}$ & $p_{11}$ & $p_{22}$ & Log Likelihood \\
\hline DGP 1a & 0.1486 & -0.0095 & 3.9869 & 0.3334 & 0.4281 & 0.9594 & 292.8283 \\
& $(0.0401)$ & $(0.0012)$ & $(0.3466)$ & $(1.3215)$ & $(0.9780)$ & $(0.6107)$ & \\
DGP 1b & -0.0806 & 14.5476 & 3.0705 & 2.7358 & 0.2398 & 0.9878 & 295.2181 \\
& $(0.0148)$ & $(10.3904)$ & $(0.4906)$ & $(0.4692)$ & $(1.7256)$ & $(0.9705)$ & \\
DGP 1c & 1.6819 & -0.1022 & 1.1125 & 0.7014 & 0.9508 & 0.4507 & 369.2432 \\
& $(0.6151)$ & $(0.0085)$ & $(0.0812)$ & $(0.1005)$ & $(2.7624)$ & $(0.2699)$ & \\
DGP 2a & 1.2373 & 0.3450 & 3.7902 & 5.3929 & 0.9502 & 0.9899 & 370.7072 \\
& $(0.4535)$ & $(0.1660)$ & $(1.8847)$ & $(0.5271)$ & $(0.5034)$ & $(3.3904)$ & \\
DGP 2b & 0.1503 & -3.2366 & 4.2380 & 2.2610 & 0.6460 & 0.9889 & 311.9050 \\
& $(0.0153)$ & $(1.5296)$ & $(0.7146)$ & $(1.2470)$ & $(1.1828)$ & $(0.9853)$ & \\
DGP 2c & -0.1319 & -3.4928 & 8.5586 & 2.1576 & 0.8337 & 0.9997 & 357.5659 \\
& $(0.0248)$ & $(7.1950)$ & $(0.8380)$ & $(1.9674)$ & $(6.2651)$ & $(5.4029)$ & \\
DGP 3a & 0.0110 & -0.1725 & 5.1182 & 0.5852 & 0.9947 & 0.5356 & 618.9616 \\
& $(0.0014)$ & $(0.0177)$ & $(0.2879)$ & $(0.6587)$ & $(1.0534)$ & $(0.8133)$ & \\
DGP 3b & 9.7536 & -0.1729 & 2.8405 & 1.7893 & 0.9918 & 0.3621 & 552.5225 \\
& $(5.4382)$ & $(0.0117)$ & $(0.4314)$ & $(0.4108)$ & $(1.0427)$ & $(1.4256)$ & \\
DGP 3c & -0.0713 & -0.3994 & 0.9445 & 0.6846 & 0.9693 & 0.5626 & 368.1439 \\
& $(0.0065)$ & $(0.0251)$ & $(0.2356)$ & $(0.1968)$ & $(0.5040)$ & $(0.8354)$ & \\
\hline Note: 1/4 & & $1 / 42$ & &
\end{tabular}

Note: $1 / \psi_{1}$ and $1 / \psi_{2}$ are the autoregressive parameters in the Markov-switching state-space model representing the two-regime counterpart of the single-regime bubble process (10). $\sigma_{\eta}$ and $\sigma_{\delta}$ are the standard deviations of the bubble and dividend processes (10) and (6). $p_{11}=\operatorname{Pr}\left\{S_{t}=1 \mid S_{t-1}=1\right\}$ and $p_{22}=\operatorname{Pr}\left\{S_{t}=2 \mid S_{t-1}=2\right\}$ are the transition probabilities of the two-regime model. Standard errors are in parentheses. 
Table 4

Tests for Markov-switching property (real-world data sets)

\begin{tabular}{lccc}
\hline & $\begin{array}{l}\text { Log Likelihood } \\
\text { Markov-switching } \\
\text { Model }\end{array}$ & $\begin{array}{l}\text { Log Likelihood } \\
\text { Linear } \\
\text { Model }\end{array}$ & $\begin{array}{l}\text { Likelihood Ratio } \\
\text { Test } \\
\text { Statistic }\end{array}$ \\
\hline USA 1871-2004 & -2105.1168 & -8998.9775 & 13787.7214 \\
USA 1871-1912 & -795.1942 & -2919.4789 & 4248.5694 \\
USA 1913-1954 & -524.8847 & -2547.8290 & 4045.8886 \\
USA 1955-2004 & -939.6068 & -3823.2714 & 5767.3292 \\
Brazil & 60.6232 & -233.2862 & 587.8188 \\
Indonesia & 83.5528 & -273.0753 & 713.2562 \\
Malaysia & 82.5899 & -525.8668 & 1216.9134 \\
Japan & -64.2102 & -1336.3819 & 2544.3434 \\
\hline
\end{tabular}


Table 5

State space model with Markov-switching (real-world data sets)

\begin{tabular}{lccccccc}
\hline & $1 / \psi_{1}$ & $1 / \psi_{2}$ & $\sigma_{\eta}$ & $\sigma_{\delta}$ & $p_{11}$ & $p_{22}$ & Log Likelihood \\
\hline USA & 0.4644 & 0.2740 & 0.0002 & 0.0452 & 0.9690 & 0.9539 & -2105.1168 \\
1871-2004 & $(0.0206)$ & $(0.0103)$ & $(0.0299)$ & $(0.0020)$ & $(0.2923)$ & $(0.2149)$ & \\
USA & 0.4565 & -0.2489 & 0.0001 & 0.0315 & 0.9424 & 0.9641 & -795.1942 \\
1871-1912 & $(0.0194)$ & $(0.0068)$ & $(0.1841)$ & $(0.0032)$ & $(0.2835)$ & $(0.2626)$ & \\
USA & 0.2776 & -0.4913 & 0.0002 & 0.0567 & 0.9733 & 0.9737 & -524.8847 \\
1913-1954 & $(0.0133)$ & $(0.0204)$ & $(0.0996)$ & $(0.0033)$ & $(0.3951)$ & $(0.4105)$ & \\
USA & 0.2711 & 0.1556 & 0.0002 & 0.0366 & 0.8518 & 0.9844 & -939.6068 \\
1955-2004 & $(0.0172)$ & $(0.0122)$ & $(0.0608)$ & $(0.0033)$ & $(0.4210)$ & $(0.5460)$ & \\
Brazil & 1.5059 & 0.6481 & 0.1489 & 0.1216 & 0.4156 & 0.9711 & 60.6232 \\
& $(0.1638)$ & $(0.1050)$ & $(0.0843)$ & $(0.0331)$ & $(1.1663)$ & $(0.9223)$ & \\
Indonesia & 2.4959 & 0.9636 & 0.0000 & 0.1839 & 0.9842 & 0.9409 & 83.5528 \\
& $(0.4362)$ & $(0.1146)$ & $(0.0775)$ & $(0.0141)$ & $(0.7606)$ & $(0.8551)$ & \\
Malaysia & 2.1041 & 0.8257 & 0.0006 & 0.1497 & 0.9747 & 0.9083 & 82.5899 \\
& $(0.3046)$ & $(0.0719)$ & $(1.2465)$ & $(0.0126)$ & $(0.5244)$ & $(0.4517)$ & \\
Japan & 2.5482 & 0.9742 & 0.0000 & 0.0994 & 0.9944 & 0.7964 & -64.2102 \\
& $(0.6712)$ & $(0.0680)$ & $(0.1467)$ & $(0.0080)$ & $(1.1556)$ & $(0.7383)$ & \\
\hline
\end{tabular}

Note: $1 / \psi_{1}$ and $1 / \psi_{2}$ are the autoregressive parameters in the Markov-switching state-space model representing the two-regime counterpart of the single-regime bubble process (10). $\sigma_{\eta}$ and $\sigma_{\delta}$ are the standard deviations of the bubble and dividend processes (10) and (6). $p_{11}=\operatorname{Pr}\left\{S_{t}=1 \mid S_{t-1}=1\right\}$ and $p_{22}=\operatorname{Pr}\left\{S_{t}=2 \mid S_{t-1}=2\right\}$ are the transition probabilities of the two-regime model. Standard errors are in parentheses. 